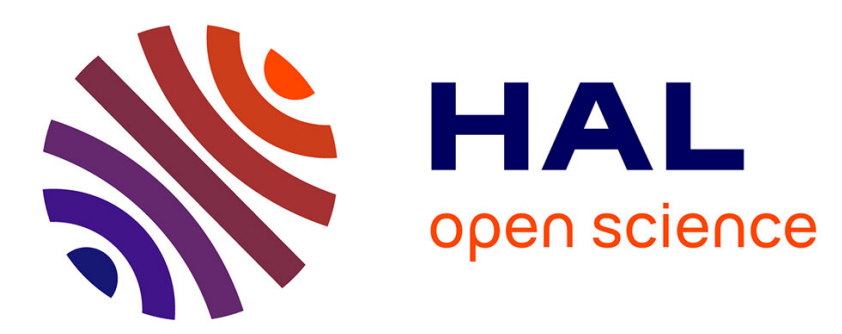

\title{
Statistical distributions of the tuning and coupling collective modes at a conical intersection using the hierarchical equations of motion
}

\author{
Etienne Mangaud, Benjamin Lasorne, Osman Atabek, Michèle
}

Desouter-Lecomte

\section{To cite this version:}

Etienne Mangaud, Benjamin Lasorne, Osman Atabek, Michèle Desouter-Lecomte. Statistical distributions of the tuning and coupling collective modes at a conical intersection using the hierarchical equations of motion. Journal of Chemical Physics, 2019, 151 (24), pp.244102. 10.1063/1.5128852 . hal-02498342

\section{HAL Id: hal-02498342 \\ https://hal.umontpellier.fr/hal-02498342}

Submitted on 22 Dec 2020

HAL is a multi-disciplinary open access archive for the deposit and dissemination of scientific research documents, whether they are published or not. The documents may come from teaching and research institutions in France or abroad, or from public or private research centers.
L'archive ouverte pluridisciplinaire HAL, est destinée au dépôt et à la diffusion de documents scientifiques de niveau recherche, publiés ou non, émanant des établissements d'enseignement et de recherche français ou étrangers, des laboratoires publics ou privés. 


\title{
Statistical distributions of the tuning and coupling collective modes at a conical intersection using the hierarchical equations of motion
}

\author{
E. Mangaud, ${ }^{1, \text { a) }}$ B. Lasorne, ${ }^{2, \text { b) }}$ O. Atabek, ${ }^{3, \text { c) }}$ and M. Desouter-Lecomte ${ }^{4, d)}$ \\ 1) Physicochimie des Electrolytes et des Nanosystèmes interfaciaux-UMR 8234 Sorbonne Université, F-75252 Paris, \\ France. \\ Laboratoire Collisions Agrégats Réactivité (IRSAMC), Université Toulouse III Paul Sabatier, UMR 5589, F-31062 Toulouse, \\ France \\ ${ }^{2)}$ Institut Charles Gerhardt UMR CNRS 5253, Université de Montpellier, ENSCM, F-34095 Montpellier, \\ France \\ ${ }^{3)}$ Institut des Sciences Moléculaires d'Orsay (ISMO) UMR CNRS 8214, Université Paris Saclay, Univ. Paris Sud, F-91405 Orsay, \\ France \\ ${ }^{4)}$ Laboratoire Chimie Physique (LCP)-CNRS,Université Paris Saclay, Univ. Paris Sud, \\ F-91405 Orsay \\ Département de Chimie, Université de Liège, Sart Tilman, B6, B-4000 Liège, Belgium, \\ France
}

We investigate the possibility of extracting the probability distribution of the effective environmental tuning and coupling modes during the non-adiabatic relaxation through a conical intersection. Dynamics are dealt with an open quantum system master equation by partitioning a multi-state electronic subsystem out of all the nuclear vibrators. This is an alternative to the more usual partition retaining the tuning and coupling modes of a conical intersection in the active subsystem coupled to a residual bath. The minimal partition of the electronic system generally leads to highly structured spectral densities for both vibrational baths and requires a strongly non-perturbative non-Markovian master equation treated here by the hierarchical equations of motion (HEOM). We extend - for a two-bath situation - the procedure proposed by Q. Shi and coworkers (J. Chem. Phys. 140, 134106 (2014)) whereby the information contained in the auxiliary HEOM matrices is exploited in order to derive the nuclear dissipative wave packet, i.e. the statistical distribution of the displacement of the two tuning and coupling collective coordinates in each electronic state and the coherence. This allows us to visualize the distribution, all along the non-adiabatic decay. We explore a large parameter space for a symmetrical conical intersection model and a symmetrical initial Franck-Condon preparation. Some parameters could be controlled by external fields while others are molecule dependent and could be designed by molecular engineering. We illustrate the relation between the strongly coupled electronic and bath dynamics together with a geometric measure of non-Markovianity.

\section{INTRODUCTION}

A conical intersection (CI) of two electronic potential energy surfaces is one of the key regions where the BornOppenheimer approximation strongly breaks down ${ }^{1-10}$. It occurs in $N$-atom systems with $N \geq 3$. According to the noncrossing rule ${ }^{11}$, two adiabatic electronic states may be energydegenerate along a $(3 N-8)$ dimensional seam when they have the same spin multiplicity or a $(3 N-7)$ one when they are of different spin multiplicity. In the former case, the other two degrees of freedom form a subspace, called branching space, in which the adiabatic potential energy surfaces appear as two cones touching at the CI (degeneracy is lifted to first order over the plane tangent to the branching space). This "funnel" is likely to induce ultrafast decay since the non-adiabatic coupling becomes infinite at the CI. The latter is thus at the core of photochemical processes in excited states and is giving rise to a wide research area, both in advanced time-dependent spectroscopy ${ }^{12-15}$ and in numerical simulations ${ }^{16-23}$. Alternatively, diabatic representations of the electronic states pro-

\footnotetext{
a)Electronic mail: etienne.mangaud@sorbonne-universite.fr

b) Electronic mail: benjamin.lasorne@umontpellier.fr

c)Electronic mail: osman.atabek@u-psud.fr

d)Electronic mail: michele.desouter-lecomte@u-psud.fr
}

vide a smoother picture of the CI in the branching space ${ }^{24,25}$. Even if a rigorous diabatisation raises issues when restricting the basis to a model made of two electronic states, as abundantly discussed in the literature ${ }^{8,26}$, a fair approximation may be derived in practice so that the diagonalization of the diabatic model yields the two adiabatic energies of interest ${ }^{23,27}$. Within a harmonic approximation, the diabatic potential energies are described by two paraboloids crossing along a line ( $3 N-7$ dimensional hyperline). The diabatic electronic coupling is often considered as varying linearly in the vicinity of conical intersections, where it is zero by construction. In the branching space, the degeneracy is lifted to first order, except at the CI point when diagonalizing the diabatic matrix provides adiabatic potential energies. The two main degrees of freedom are the tuning mode (TM), $Q_{t}$, along which the diabatic energy gap varies to first order, and the coupling mode $(\mathrm{CM}), Q_{c}$, that induces the linear electronic coupling ${ }^{28-30}$.

Simulating excited-state non-adiabatic dynamics in extended systems remains a challenge and an active topic. This requires a compromise between the computational scaling and the need to explore accurately, and in real time, many environmental degrees of freedom at any temperature since dissipation is responsible for electronic relaxation. Numerical methods may be classified according to the explicit or implicit description of the environmental degrees of freedom. The first strategy involves an explicit description of the elec- 
Adiabatic

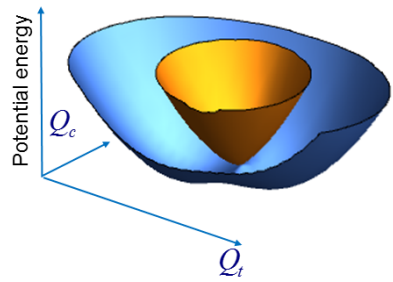

Diabatic

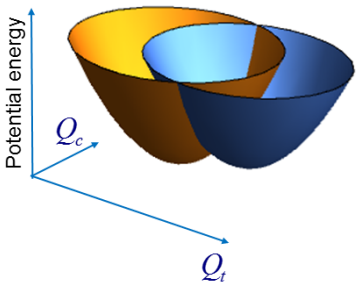

FIG. 1. Left panel: Schematic view of a conical intersection in the adiabatic representation along the branching space of the tuning $Q_{t}$ and coupling $Q_{c}$ modes. Right panel: the diabatic representation in which the electronic coupling is varying linearly along the coupling mode.

tronic system and of a large sample of vibrators. It has been addressed by various strategies ranging from fully quantum methods to semi-classical approaches, for instance multiconfiguration time-dependent Hartree (MCTDH) ${ }^{19,31}$, multilayer-MCTDH ${ }^{32}$, variational approaches with Gaussian basis sets (ab initio multiple spawning $\mathrm{AIMS}^{33,34}$, direct-dynamics variational multiconfiguration Gaussian DD-vmCG) ${ }^{35}$, tensorial matrix product approaches ${ }^{36}$ or mixed quantum-classical trajectories $^{37}$, semiclassical surface hopping ${ }^{38,39}$. Full quantum methods based on wave functions suffer from the scaling and from the difficulty of simulating high temperature. On the other hand, semi-classical strategies may lead to a questionable description of the quantum noise or of tunneling effect. An alternative description is dissipative dynamics ${ }^{40}$, which treats only implicitly the environment at any temperature with statistical Schrödinger equations ${ }^{41-43}$, multi-state quantum Fokker-Planck equation (MSQFPE) ${ }^{44-47}$, or master equations in Liouville space. Observables related to the selected active subspace can thus be obtained exactly in the framework of the harmonic baths with linear couplings by non-perturbative methods such as the "hierarchical equations of motion" (HEOM) formalism ${ }^{48,49}$.

The partitioning between an active subsystem and a thermal bath determines not only the coupling strength and therefore the computational scaling and the tractable Markovian or nonMarkovian master equations but also the accuracy of the information about some environmental modes. In a two-electronic state case, the two main strategies are the spin-boson or "star model" and the effective reaction coordinate model. In the first case (denoted two-level system TLS), the electronic states are coupled to the ensemble of environmental modes and in the second, one or more primary effective coordinates are retained in the active system and are coupled to a residual bath. In the CI situation, the TM and CM modes are included in the system denoted two-level system two-coordinate model (TLSTC). The relative canonical transformation of the vibrational modes has been discussed for a long time ${ }^{50-56}$ and in particular the relation between the corresponding spectral densities has been given in a seminal paper about electron transfer ${ }^{57}$. This has given rise to an abundant literature using one or the other partitioning with different dynamical treatments ${ }^{58-68}$.
The particular CI case has been frequently dealt for instance with a perturbative Markovian Redfield approach ${ }^{69}$, time nonlocal second-order master equation ${ }^{70}$, Wigner distribution ${ }^{45}$, hybrid quantum master equation ${ }^{71}$, and recently beyond perturbative regime by $\operatorname{HEOM}^{72,73}$. One of the advantages is the accurate monitoring of the dissipative wave packet in the branching space that can be mapped by time and frequency resolved fluorescence spectroscopy ${ }^{73}$. In particular, it enables to get information about the vibrational coherence that influences the dynamics through the CI. However, the dimension of the basis set for a two-dimension two- or many-electronicstate system may become prohibitive for HEOM simulations since all the so-called auxiliary matrices of the corresponding coupled equations have the same dimension as that of the system. An interesting alternative has been suggested recently ${ }^{74,75}$. In this approach, the system describes only two (or more) electronic states (two-level system TLS) while the effective TM and CM are included in two highly structured baths. In most of the cases, this requires non-perturbative, non-Markovian treatments and, therefore, HEOM with a high level of hierarchy. The number of auxiliary matrices may be very large but they have a small dimension (only two by two in the TLS). This scaling is thus favorable mainly when several electronic states are considered ${ }^{75}$.

The main point addressed in the present work is to show that HEOM techniques enable us to access information about environmental processes even in the minimal TLS partition. We define tools able to extract the probability distribution of the effective modes of the structured spectral densities in real time from the auxiliary matrices. In this way, we can visualize the interplay between the electronic transition and the vibrational motion leading to a strong restructuring of the statistical distribution, which does not remain a passive unperturbed Gaussian distribution during the non-adiabatic evolution. We extend for two baths the interesting strategy proposed by Shi et $a l .^{76,77}$ so as to extract this statistical distribution of the collective TM and CM from the auxiliary matrices. The time evolution of the distribution, in particular the average position or the distortion of the initial Gaussian profile before reaching the asymptotic regime provide valuable information about the damped vibrational motion at any temperature.

We illustrate the general trends induced by typical changes made on the system or on the environmental spectral densities even if it is not possible to entirely explore the large parameter space. Some parameters related to the system could be controlled by interaction with laser fields such as the energy gap and the position of the CI by a Stark shift ${ }^{78-81}$ or the preparation of the initial wave packet by modifying the initial superposition of the electronic states during the ultra fast excitation. Some parameters are also molecule-dependent, and exploring the main behaviors is a timely issue in molecular engineering to design new generations of quantum-assisted molecular systems for energy transfer ${ }^{82}$. Indeed it is possible to vary the parameters entering the model Hamiltonian (potential energies and couplings) according to the chemical nature of the chromophore within the molecular system. They will also be sensitive to the presence of covalent bridges and substituents (intramolecular interactions) and to the surrounding medium 
(intermolecular interactions with a cage, a protein, or a solvent). For instance, a crossing between a locally excited state and a charge-transfer state can be displaced both in energy and position upon playing with the stabilisation or destabilisation of the charge separation with different types of electroactive substituents or with solvents of various polarities. The relative positions, energies, and frequencies of the two minima will also be affected by the extent of the reorganization of the bonding pattern induced by the electronic excitation: a large Stokes shift between absorption and emission spectra will be the signature of a strong rearrangement. One can choose a system in which one electronic state has similar parameters with respect to the ground state, while the other is strongly shifted. This is likely to be the case when a state is very bright and the other is dark. Finally, modulating the intrastate coupling can be achieved upon changing the nature of the bridge between the donor and the acceptor in a system experiencing charge or excitation energy transfer between two sites. Through-bond and through-space processes should correspond to strong and weak couplings, respectively.

The paper is organized as follows. In Sec.IIA we define the main parameters the TLS and TLS-TC models and their relations. The spectral densities of both models and the correlation functions are given in Sec.IIB. The methodology to extract the statistical distribution of the baths is summarized in Sec.IIC while the HEOM equations for two uncorrelated baths are given in the Appendix. Sec.IID presents one possible non-Markovianity signature used in this work. Results of numerical simulations are presented in Sec.III where we illustrate the interplay between TM and CM collective modes and the dissipative electronic wave packet around a CI in a TLS model.

\section{METHODS}

\section{A. System-environment partitioning}

We consider three electronic states with a ground state $|0\rangle$ decoupled from a doublet of coupled excited diabatic states $|1\rangle$ and $|2\rangle$. In our model, the ground state $|0\rangle$ and the first excited state $|1\rangle$ have the same equilibrium positions but the second one $|2\rangle$ is spatially displaced. By restricting the electronic basis set to two excited states, the full vibronic Hamiltonian in the diabatic representation reads

$$
H=\sum_{n=1}^{2}|n\rangle H_{n}\langle n|+\left(|1\rangle H_{12}\langle 2|+\text { h.c. }\right)
$$

where h.c denotes Hermitian conjugation $H_{n}$ is the vibrational Hamiltonian associated with each electronic state $|n\rangle$ and treated within a harmonic level of approximation. By assuming that the frequencies are the same in both states but the equilibrium positions are different, and by separating the $N_{t}$ modes (label $t$ standing for tuning) that tune the energy gap from the $N_{c}$ modes $(c)$ that induce a variation of the electronic

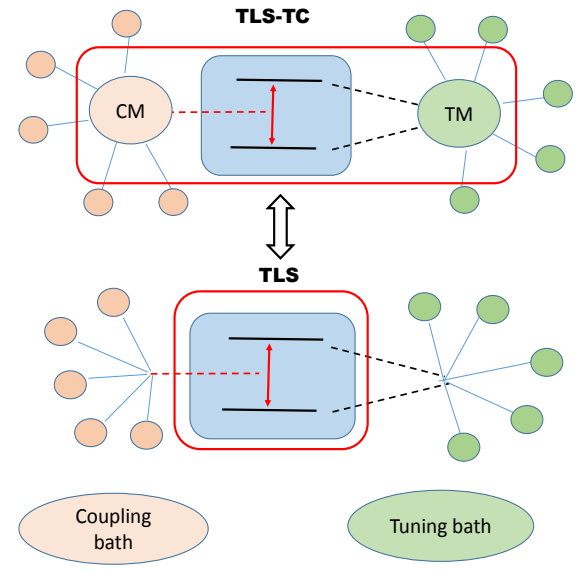

FIG. 2. Upper panel: Schematic representation of the TLS-TC model in which the system includes two two-dimensional vibrational Hamiltonians associated to two diabatic electronic states while the environment is formed by modes coupled to the effective TM and CM of a CI. Lower panel: the TLS in which the electronic energy gap is coupled to a tuning bath of oscillators and the electronic coupling to a coupling bath.

coupling, $H_{n}$ reads in mass-weighted coordinates

$$
\begin{aligned}
H_{n}= & \varepsilon_{n}+1 / 2 \sum_{k=1}^{N_{t}}\left(p_{t, k}^{2}+\omega_{t, k}^{2}\left(q_{t, k}-q_{t 0, k}^{(n)}\right)^{2}\right) \\
& +1 / 2 \sum_{k=1}^{N_{c}}\left(p_{c, k}^{2}+\omega_{c, k}^{2}\left(q_{c, k}-q_{c 0, k}^{(n)}\right)^{2}\right) .
\end{aligned}
$$

The reference point that defines the equilibrium geometry of the initial vibrational bath for the partition is the equilibrium position of the ground state. It is chosen as $q_{t, k}=0$ and $q_{c, k}=$ 0 . The equilibrium positions of the tuning modes are also equal to zero in the first electronic state, $q_{t 0, k}^{(1)}=0$, and those of the second state will be denoted $q_{t 0, k}^{(2)}=q_{0 k}$. The equilibrium positions of the coupling modes are the same in both states and equal to zero $q_{c 0, k}^{(1)}=q_{c 0, k}^{(2)}=0$. The electronic coupling is varying linearly from the CI position,

$$
H_{12}=\sum_{k=1}^{N_{c}} c_{k}\left(q_{c, k}-q_{C I, k}\right) .
$$

We consider a symmetrical case for which the CI position corresponds to the equilibrium positions of the coupling modes, $q_{C I, k}=q_{c 0, k}=0$.

We now summarize the operational relations of the two TLS or TLS-TC partitions for the CI model. They are illustrated in Fig.2.

\section{TLS partition}

When partitioning so as to isolate the electronic subsystem, the full Hamiltonian adapted to the CI case is recast as $H=H_{S}+H_{B t}+H_{B c}+S_{t} B_{t}+S_{c} B_{c}$. According to the chosen reference point the system Hamiltonian in the basis set $|1\rangle,|2\rangle$ 
is :

$$
H_{S}=\frac{\Delta}{2} \sigma_{z}+\frac{1}{2}\left(\mathbf{I}-\sigma_{z}\right) \lambda_{t}+W_{12} \sigma_{x}
$$

where $\sigma$ are the Pauli matrices, I the unity matrix, $\Delta=\varepsilon_{2}-\varepsilon_{1}$ is the energy gap between the two minima of the diabatic potentials, $W_{12}$ is the electronic coupling at the reference point. When it is chosen at the equilibrium position of the coupling modes, $q_{c 0, k}$, one has $W_{12}=0 . \lambda_{t}=\sum_{k} \omega_{t, k}^{2} q_{0 k}^{2} / 2$ is the renormalization energy (also called reorganization energy) of the tuning bath. With the chosen reference point, it is added only to the energy of state $|2\rangle$. The two baths are $H_{B t}=$ $\frac{1}{2} \sum_{i=1}^{N_{t}}\left(p_{t, i}^{2}+\omega_{t, i}^{2} q_{t, i}^{2}\right)$ and $H_{B c}=\frac{1}{2} \sum_{i=1}^{N_{c}}\left(p_{c, i}^{2}+\omega_{c, i}^{2} q_{c, i}^{2}\right)$. The system-bath interaction involves collective coordinates $B_{t}=$ $\sum_{k=1}^{N_{t}} d_{k} q_{t, k}$ with $d_{k}=q_{t 0, k} \omega_{t, k}^{2}$ and $B_{c}=\sum_{k=1}^{N_{c}} c_{k} q_{c, k}$ ( $c_{k}$ being defined through Eq.(3)). $B_{t}$ is diagonally coupled to the electronic system through the operator $S_{t}=\frac{1}{2}\left(\sigma_{z}-\mathbf{I}\right)$ (since only state $|2\rangle$ is coupled to the bath due to the choice made for the reference point) while $B_{c}$ is off-diagonally coupled by the operator $S_{c}=\sigma_{x}$.

\section{TLS-TC partition}

In order to incorporate the largest part of the vibronic coupling into the enlarged system, a unitary transformation of the bath oscillators is made to separate in each case the effective coordinate that captures the whole coupling $51-56,60-62,83$, $\left\{q_{t, i}\right\} \Rightarrow\left\{Q_{t}, Q_{j}\right\} \quad j=2, N_{t}$ and $\left\{q_{c, i}\right\} \Rightarrow$ $\left\{Q_{c}, Q^{\prime}{ }_{j}\right\} \quad j=2, N_{c}$ where $Q_{t}=\frac{1}{D_{t}} \sum_{k=1}^{N_{t}} d_{k} q_{t, k}$ with $D_{t}^{2}=$ $\sum_{j=1}^{N_{t}} d_{j}^{2}$ and $Q_{c}=\frac{1}{D_{c}} \sum_{k=1}^{N_{c}} c_{k} q_{c, k}$ with $D_{c}^{2}=\sum_{j=1}^{N_{c}} c_{j}^{2}$. The unitary transformation is built so that the residual modes are coupled to the effective one but are not coupled with each other. The transformed Hamiltonian (Eq.1) is by accounting for the reference point

$$
\tilde{H}=\sum_{n=1}^{2}|n\rangle \tilde{H}_{n}\langle n|+\left(|1\rangle \tilde{W}_{12}\langle 2|+\text { h.c. }\right)
$$

with

$$
\begin{gathered}
\tilde{H}_{1}=\varepsilon_{1}+1 / 2\left(P_{t}^{2}+\Omega_{t}^{2} Q_{t}^{2}\right)+1 / 2\left(P_{c}^{2}+\Omega_{c}^{2} Q_{c}^{2}\right)+\tilde{H}_{B} \\
\tilde{H}_{2}=\varepsilon_{2}+\lambda_{2}+1 / 2\left(P_{t}^{2}+\Omega_{t}^{2} Q_{t}^{2}\right)-D_{t} Q_{t}+1 / 2\left(P_{c}^{2}+\Omega_{c}^{2} Q_{c}^{2}\right)+\tilde{H}_{B} \\
\tilde{H}_{B}=1 / 2 \sum_{k=2}^{N_{t}}\left(P_{k}^{2}+\tilde{\Omega}_{k}^{2}\left(Q_{k}+\tilde{d}_{k} Q_{t} / \tilde{\Omega}_{k}^{2}\right)^{2}\right) \\
+1 / 2 \sum_{k=2}^{N_{c}}\left(P_{k}^{\prime 2}+\tilde{\Omega}_{k}^{\prime 2}\left(Q_{k}+\tilde{c}_{k} Q_{c} / \tilde{\Omega}_{k}^{\prime 2}\right)^{2}\right) \\
\tilde{H}_{12}=D_{c} Q_{c}
\end{gathered}
$$

The TLS-TC partitioning retains in the system Hamiltonian $\tilde{H}_{S}$ all the terms of $\tilde{H}$ related to the two-dimensional subspace and the counter-terms coming from $Q_{t}^{2}$ and $Q_{c}^{2}$ in $\tilde{H}_{B}{ }^{66,74,84}$.
For the TM, the coupling constant $D_{t}$ determines the displacement $D_{t} / \Omega_{t}^{2}$ between the equilibrium position of the two effective diabatic parabolas while the coupling $D_{c}$ fixes the slope of the linear electronic coupling in the diabatic representation. The residual tuning and coupling baths gather all the new oscillators $Q_{j}$ or $Q_{j}^{\prime}$ and the system-bath couplings now involve cross terms $\tilde{d}_{j} Q_{t} Q_{j}$ or $\tilde{c}_{j} Q_{c} Q_{j}^{\prime}$, respectively. The effective frequencies resulting from the transformed Hessian matrix ${ }^{54}$ are given by $\Omega_{t}^{2}=\frac{1}{D_{t}^{2}} \sum_{i=1}^{N_{t}} d_{i}{ }^{2} \omega_{t, i}^{2}$ and $\Omega_{c}^{2}=\frac{1}{D_{c}^{2}} \sum_{i=1}^{N_{c}} c_{i}^{2} \omega_{c, i}^{2}$.

\section{B. Spectral densities and correlation functions}

In open quantum systems, the system-bath interaction is characterized by the spectral density, which has a generic expression:

$$
J(\omega)=\frac{\pi}{2} \sum_{k} f_{k}^{2} \omega_{k}^{-1} \delta\left(\omega-\omega_{k}\right)
$$

where $f_{k}^{2}$ is the strength of the linear coupling at each frequency. In the TLS, the spectral densities of the tuning and coupling baths are $J_{t / c}(\omega)$ with $f_{k}=d_{k}$ and $f_{k}=c_{k}$, respectively, while the spectral densities of the residual baths in the TLS-TC are $J_{t / c}^{r e s}(\omega)$ with $f_{k}=\tilde{d}_{k}$ and $f_{k}=\tilde{c}_{k}$. The spectral density has been introduced here in a discrete representation as it should be the case when the displacements come from an $a b$ initio normal mode analysis. When the frequency density is high, one may switch to the continuous representation by broadening the delta distribution by a Lorentzian function $\delta\left(\omega-\omega_{k}\right) \rightarrow \frac{1}{\pi} \frac{\Gamma}{\left(\omega-\omega_{k}\right)^{2}+\Gamma^{2}}$ as suggested for instance in $\operatorname{refs}^{85,86}$. Note that a continous spectral density is directly provided by experiments or by molecular dynamics simulations giving the correlation of the energy gap ${ }^{87,88}$. A discretization taking into account the frequency density is then used for simulations of MCTDH type ${ }^{89}$.

The temperature-dependent spectral density $\tilde{C}(\omega)=J(\omega)\left(e^{\beta \omega}-1\right)^{-1}$ with $\beta=1 / k_{B} T$ is the Fourier transform of the correlation function of the collective mode $C(t)=\operatorname{Tr}_{B}\left[B(t) B(0) \rho_{B}^{e q}\right]$ where $\rho_{B}^{e q}=\exp \left(-\beta H_{B}\right) / \operatorname{Tr}_{B}\left[\exp \left(-\beta H_{B}\right)\right]$ is the Boltzmann equilibrium density matrix of the bath and $B(t)=\exp \left(i H_{B} t\right) B \exp \left(-i H_{B} t\right)($ with $\hbar=1$ a.u. $)$

$$
C(t)=1 / \pi \int_{-\infty}^{+\infty} d \omega \tilde{C}(\omega) e^{i \omega t}
$$

The relation between the spectral density $J^{\text {res }}(\omega)$ of the one-effective mode two-level model with $J(\omega)$ of the twolevel model (spin-boson model) has been derived in ref. ${ }^{57}$. When $J^{r e s}(\omega)$ is Ohmic with a friction coefficient $\eta$ given by the slope for $\omega=0$, the spin-boson spectral density is a Lorentzian function whose width is proportional to this friction. A small friction in one-effective mode model leads to a highly peaked $J(\omega)$ in TLS model. 
Here, we assume Lorentzian functions for the spectral densities of the tuning and coupling baths given by the TannorMeier expression ${ }^{90}$

$$
J_{t / c}(\omega)=\frac{p_{t / c} \omega}{\left[\left(\omega+\omega_{t / c}\right)^{2}+\Gamma_{t / c}^{2}\right]\left[\left(\omega-\omega_{t / c}\right)^{2}+\Gamma_{t / c}^{2}\right]}
$$

where $\omega_{t / c}$ gives the peak position, $\Gamma_{t / c}$ is the width and $p_{t / c}$ is a parameter fixing the coupling strength. The corresponding residual spectral density of the TLS-TC model is computed by the following relation, $62,65-68,91$

$$
J_{t / c}^{r e s}(\omega)=\frac{D_{t / c}^{2} J_{t / c}(\omega)}{J_{t / c}^{2}(\omega)+W_{0, t / c}^{2}(\omega)}
$$

where

$$
W_{0, t / c}(\omega)=\frac{1}{\pi} P V \int_{-\infty}^{+\infty} d \omega^{\prime} \frac{J_{t, c}\left(\omega^{\prime}\right)}{\omega^{\prime}-\omega}
$$

with $P V$ the principal value distribution of the Hilbert transform of the TLS spectral density. By using Eq.(14), the residual spectral density is an Ohmic function with a friction coefficient $\eta_{t / c}=2 \Gamma_{t / c}$. With this parametrization (Eq.(13)), the correlation function (Eq.(12)) takes the form of a sum of decreasing complex exponentials well adapted for applying the HEOM algorithm, ${ }^{77}$

$$
C_{t / c}(t)=\sum_{k=1}^{n_{c o r, t / c}} \alpha_{t / c, k} e^{i \gamma_{t / c, k} t}
$$

As shown in ref. ${ }^{92}$, the complex conjugate may be expressed with the same $\gamma_{t / c, k}$ but modified $\alpha_{t / c, k}$ coefficients

$$
C_{t / c}^{*}(t)=\sum_{k=1}^{n_{c o r}, / c} \tilde{\alpha}_{t / c, k} e^{i \gamma_{t / c, k} t}
$$

$n_{c o r, t / c}$ contains two terms for each two-pole Lorentzian function plus a number of Matsubara terms corresponding to the poles of the Bose function. Analytical expressions of $\alpha, \tilde{\alpha}$, and $\gamma$ are given in refs. ${ }^{65,92}$.

In the continuous limit, the renormalization energy $\lambda_{t / c}$, the effective mode frequencies $\Omega_{t / c}$, and the coupling constant $D_{t / c} \mathrm{read}$

$$
\begin{gathered}
\lambda_{t / c}=1 / \pi \int_{0}^{\infty} d \omega J_{t / c}(\omega) \omega^{-1} \\
D_{t / c}^{2}=\frac{2}{\pi} \int_{0}^{+\infty} d \omega J_{t / c}(\omega) \omega \\
\Omega_{t / c}^{2}=\frac{2}{\pi D_{t / c}^{2}} \int_{0}^{+\infty} d \omega J_{t / c}(\omega) \omega^{3} .
\end{gathered}
$$

The linear couplings of the TLS-TC model may be related to the renormalization energies by the relation ${ }^{68,74}$ given here in mass weighted coordinates

$$
D_{t / c}=\sqrt{2 \lambda_{t / c}} \Omega_{t / c}
$$

\section{Statistical distribution of $B_{t / c}$}

The hierarchical equations of motion are one of the reference dynamical methods for open quantum systems modelled with a bath of harmonic oscillators inducing stochastic modulation of the diabatic energy gap and of the electronic coupling. The equations giving the evolution of the reduced density matrix, $\rho(t)=\operatorname{Tr}_{B}\left[\rho_{\text {tot }}(t)\right]$, were originally derived for a Drude-Lorentz spectral density in the high-temperature limit from the Kubo stochastic Liouville equation ${ }^{93}$ and the Feynman-Vernon influence functional formalism ${ }^{48,49}$. They have been extended for lower temperature ${ }^{94,95}$ and for fermion and boson grand-canonical bath ensembles ${ }^{96}$. The efficiency of the algorithm relies on the expansion of the correlation function $C(t)$ as a sum of $n_{\text {cor }}$ complex exponential functions associated to pseudo decay modes of decay. Different expansions have been proposed ${ }^{77}$ among which the Tannor-Meier one leading to Eqs.16 and 17 90,92 , the Padé approximation ${ }^{97}$, and the Fano spectrum decomposition scheme ${ }^{98}$. A direct decomposition of the bath correlation function based on Chebyshev polynomials and Bessel functions has also been proposed leading to the so called C-HEOM scheme ${ }^{99}$.

The method has been applied to several processes, for instance to simulate excitation energy transfer in photosynthetic light harvesting complexes ${ }^{100-104}$, electron transfer ${ }^{64,84,105,106}$, quantum transport ${ }^{107}$, heat transport $^{108}$, charge separation dynamics ${ }^{109}$. We assume here an initial factorization between the system and the bath, $\rho_{\text {tot }}(0)=\rho(0) \rho_{B}^{e q}$ valid for an ultrafast electronic transition (vertical FranckCondon transition $)^{100}$. The equilibrium initial bath is then the Boltzmann distribution of vibrational states in the ground electronic state projected in the bright excited state, and this initial condition is valid when the vibrational relaxation is not faster than the electronic dynamics. Initial correlation could be taken into account with different strategies ${ }^{105,110,111}$. The operational equations adapted for two uncorrelated baths and to the chosen expansion of $C(t)$ are summarized in the Appendix. Here, we focus on the way the bath information is extracted from the auxiliary matrices in the two-bath case (i.e. the CI TLS model) by generalizing the methodology originally derived by Shi et al.$^{76,77}$ and recently illustrated in a single bath and three-state case ${ }^{112}$.

The auxiliary operators are matrices of the same dimension as the system density matrix. They are denoted by a collective index $\mathbf{n}=\left\{\mathbf{n}_{t}, \mathbf{n}_{c}\right\}$ giving the excitation number in the pseudo modes of each correlation function: $\mathbf{n}_{j}=\left\{n_{1, j}, \cdots, n_{n_{\text {cor }}, j}\right\}$ where $j=t, c$ and $n_{k}$ is the quantum number of the $k^{t h}$ pseudo mode. The system density matrix $\rho(t)$ is given by the top row, i.e. $\mathbf{n}=\{0, \cdots, 0\}$ hence $\rho(t)=\rho_{I}(t)=\rho_{\{0, \cdots, 0\}}(t)$. The level of the hierarchy is equal to the sum of all the quantum numbers of the two baths. For each bath, one can also define the partial level to which belongs a given auxiliary matrix by summing the quantum numbers of only $\mathbf{n}_{t}$ or $\mathbf{n}_{c}$. Extending the Shi et al methodology ${ }^{76}$, one may obtain the high-order moments of the collective bath coordinate $B_{t / c}$ in each electronic state from an operator in the system subspace defined 
for the $n^{\text {th }}$ order by

$$
\mathbf{X}_{t / c}^{(n)}(t)=\operatorname{Tr}_{B_{t / c}}\left[\mathbf{B}_{t / c}^{n} \rho_{t o t}(t)\right]
$$

In particular, each diagonal element $X_{t / c, \alpha \alpha}$ of $\mathbf{X}_{t / c}^{(1)}$ gives the expectation value of the collective coordinate $B_{t / c}$ in the corresponding electronic state $\alpha$. This first moment can be obtained from the matrices contributing to the partial first level for each bath,

$$
\mathbf{X}_{t / c}^{(1)}(t)=-\sum_{\mathbf{n}} \rho_{\mathbf{n}}(t)
$$

where the sum runs over all the collective index $\mathbf{n}$ for which $\sum_{k} n_{k, i}=1$ and $\sum_{k} n_{k, j}=0$ with $i=t / c$ and $j=c / t$ respectively. Each diagonal element $X_{t / c, \alpha \alpha}$ may be renormalized to have the dimension of the effective coordinate $Q_{t / c}$. These diagonal elements $X_{j, \alpha \alpha} / D_{j}$ with $j=t, c$ will be simply denoted $X_{t / c} / D_{t / c}$ without notation of the electronic state when the latter is clearly specified. This quantity may be compared to the average position $\left\langle Q_{t / c}(t)\right\rangle$ of the effective mode computed in the present work in an undamped two-dimensional effective space (i.e., with the system Hamiltonian $\tilde{H}_{S}$. The first moment already provides interesting information about the TM vibrational relaxation and the evolution towards equilibrium. However, in the particular case of a CI with a symmetric preparation in the bright state, the average of the $\mathrm{CM}$ vanishes and the first moment is not sufficient to analyze the relaxation.

Deeper insight is provided by the full statistical distribution of the collective modes $B_{t / c}$ or of the collective coordinate $x_{t / c}=B_{t / c} / D_{t / c}$ in each electronic state. We first discuss the projection on the collective coordinates $P_{\alpha}\left(x_{t / c}, t\right)$ where $\alpha$ denotes the electronic state. $P_{\alpha}\left(x_{t / c}, t\right)$ may be compared to the projection of the two-dimensional undamped wave packet in each electronic state

$$
P_{\alpha}\left(Q_{t / c}, t\right)=\int d Q_{c / t}\left|\Psi_{\alpha}\left(Q_{t}, Q_{c}, t\right)\right|^{2}
$$

$P_{\alpha}\left(x_{t / c}, t\right)$ is expanded in a Hermite polynomial basis set,

$$
P_{\alpha}\left(x_{j}, t\right)=\sum_{n} a_{\alpha, j, n}(t) \Phi_{n}\left(x_{j}\right)
$$

with

$$
\Phi_{n}\left(x_{j}\right)=\frac{D_{j}}{\sqrt{2 \pi C_{j}(0)} \sqrt{2^{n} n !}} H_{n}\left(D_{j} x_{j} / \sqrt{2 C_{j}(0)}\right) e^{-\frac{D_{j} x^{2}}{2 C_{j}(0)}}
$$

where $j=t / c, H_{n}$ is the Hermite polynomial of order $n$ and $C_{j}(0)$ is the initial value of the correlation function (Eq.(16)), i.e. the integral of the temperature-dependent spectral density $\tilde{C}_{j}(\omega)$. The expression is adapted from refs. ${ }^{76,77}$ to ensure a correct normalization of the distribution and to map from $B_{j}$ to coordinate $x_{j}=B_{j} / D_{j}$. The procedure may be understood from an early interpretation of the auxiliary matrices as time dependent coefficients of an expansion of the stochastic Liouville equation ${ }^{93}$ in a basis set of the eigenvectors of the corresponding relaxation operator ${ }^{98,113}$. The ratio $D_{j}^{2} / C_{j}(0)$ in $P\left(x_{j}\right)$ does not depend on the coupling strength. For each electronic state $\alpha$ the coefficient $a_{\alpha, j, n}(t)$ is the diagonal element of a matrix built from the auxiliary matrices of partial level $n$ for the bath $j$

$$
\mathbf{A}_{j, n}(t)=\frac{(-1)^{n}}{\sqrt{n ! C_{j}^{n}(0)}} \sum_{\mathbf{n}} \frac{n !}{\prod_{k} n_{k, j} !} \rho_{\mathbf{n}}(t) \quad \sum_{k} n_{k, j}=n
$$

where $j=t, c$. The sum runs over the index $\mathbf{n}$ for which the partial level of bath $j$ is equal to $n$ and that of the other bath is equal to zero. It is worth noting that a similar treatment has been proposed to extract the distribution of the moment conjugated to the collective mode in order to calculate thermal fluxes ${ }^{108}$.

We now generalize the procedure to get the more informative two-dimensional distribution $P_{\alpha}\left(x_{t}, x_{c}, t\right)$ that may be compared to the square modulus of the undamped two-dimensional wave packet $\left|\Psi_{\alpha}\left(Q_{t}, Q_{c}, t\right)\right|^{2}$. The coherence $\chi_{\alpha, \beta}\left(x_{t}, x_{c}, t\right)$ corresponds to the cross product of the wave packets in each electronic state $\xi_{\alpha \beta}\left(Q_{t}, Q_{c}, t\right)=$ $\Psi_{\alpha}^{*}\left(Q_{t}, Q_{c}, t\right) \Psi_{\beta}\left(Q_{t}, Q_{c}, t\right)$. We build an array of matrices of the same dimension as $\rho(t)$

$$
\begin{gathered}
\mathbf{M}_{n, m}(t)=\frac{(-1)^{n} D_{t}}{\sqrt{n ! C_{t}^{n}(0)}} \frac{(-1)^{m} D_{c}}{\sqrt{m ! C_{c}^{m}(0)}} \sum_{\mathbf{n}_{t}} \frac{n !}{\prod_{k} n_{k, t} !} \sum_{\mathbf{n}_{c}} \frac{m !}{\prod_{k} n_{k, c} !} \rho_{\mathbf{n}_{t}, \mathbf{n}_{c}}(t) \\
\sum_{k} n_{k, t}=n \quad \sum_{k} n_{k, c}=m .
\end{gathered}
$$

The two-dimensional distribution $P_{\alpha}\left(x_{t}, x_{c}, t\right)$ in the electronic state $\alpha$ is then given by

$$
P_{\alpha}\left(x_{t}, x_{c}, t\right)=\sum_{n, m} M_{n, m}^{\alpha \alpha}(t) \Phi_{n}\left(x_{t}\right) \Phi_{m}\left(x_{c}\right)
$$

where $M_{n, m}^{\alpha \alpha}$ is the corresponding diagonal element. Moreover, one can extract information about the electronic coherence as a function of the two coordinates from the off diagonal elements:

$$
\chi_{\alpha \beta}\left(x_{t}, x_{c}, t\right)=\sum_{n m} M_{n, m}^{\alpha \beta}(t) \Phi_{n}\left(x_{t}\right) \Phi_{m}\left(x_{c}\right) .
$$

The quantities extracted from the auxiliary matrices are here related to the diabatic description of the dynamics. The adiabatic electronic coherence during relaxation through a $\mathrm{CI}$ has been recently computed by the MSQFPE method ${ }^{45}$.

The distribution $P_{\alpha}\left(x_{t}, x_{c}, t\right)$ and the coherence $\chi_{\alpha, \beta}\left(x_{t}, x_{c}, t\right)$ are computed here in the diabatic representation. Transforming to the adiabatic representation is possible by using the parameters of the corresponding two-dimensional TLS-TC model. At each point $\left(x_{t}, x_{c}\right)$ one may compute the real orthogonal diabatic-to-adiabatic transformation matrix by diagonalizing the local two-by-two diabatic electronic matrix. The adiabatic electronic states are then given by :

$$
\psi_{k}^{a d}\left(x_{t}, x_{c}\right)=\sum_{k^{\prime}=1,2} S\left(x_{t}, x_{c}\right) \psi_{k^{\prime}}^{d i a}\left(x_{t}, x_{c}\right) .
$$

As the distribution $P_{\alpha}\left(x_{t}, x_{c}, t\right)$ maps the product $\psi_{\alpha}^{* d i a}\left(x_{t}, x_{c}\right) \psi_{\alpha}^{\text {dia }}\left(x_{t}, x_{c}\right)$, one easily gets at each point 


$$
\begin{aligned}
\left(x_{t}, x_{c}\right): & \\
& P_{1}^{a d}=S_{11}^{2} P_{1}^{d i a}+S_{21}^{2} P_{2}^{d i a}+2 S_{11} S_{21} \Re_{e}\left(\chi_{12}\right) \\
P_{2}^{a d} & =S_{12}^{2} P_{1}^{d i a}+S_{22}^{2} P_{2}^{\text {dia }}+2 S_{12} S_{22} \Re_{e}\left(\chi_{12}\right)
\end{aligned}
$$

\section{Non-Markovianity signature}

Definition and measure of non-Markovianity has given rise to an abundant literature in the last decade ${ }^{114,115}$. The first crude estimation is based on the comparison between a characteristic timescale of the system dynamics and the bath correlation time. Assuming a delta-function for $C(t)$ and therefore a flat spectral density (white noise) leads to memoryless dynamics which may be treated by Markovian Lindblad or Redfield approaches (at least in perturbative regime) ${ }^{116}$. In the TLS model of a CI, the spectral density is expected to be strongly structured, thus leading to long correlation times so that CI electronic dynamics described by this partition should be strongly non-Markovian. It is commonly said that nonMarkovianity means back-flow from the bath to the system ${ }^{40}$. In the electronic dynamics, this involves a boost of electronic average energy from the vibrational modes, in other words a non-monotonic electronic relaxation. We use here a nonMarkovianity witness given by the volume of the accessible states in the Bloch sphere ${ }^{117}$. This geometric measure is not a very sensitive method to detect the non-Markovianity ${ }^{118}$ mainly at long times but it is easy to compute and is independent of the initial conditions of the system.

For an $N$-level system, HEOM defines a non-Markovian dynamical map, $\rho(t)=\phi_{t}[\rho(0)]$, that may be expressed in a basis set of $N^{2}$ operators in the Liouville space including the identity $G_{0}=I / \sqrt{N}$ and the $N^{2}-1$ generators of $S U(N)$, $G_{i}\left(i=1, \ldots, N^{2}-1\right)^{119,120}$. These generators are the Pauli spin matrices when $N=2$. In matrix form the map reads $F_{m, n}(t)=\operatorname{Tr}\left(G_{m} \phi_{t}\left[G_{n}\right]\right)$ and the volume of accessible states is obtained from the determinant of the matrix of the map,

$$
V(t)=\operatorname{det}(\mathbf{F}(t)) .
$$

A non-monotonic decrease of this volume is a signature of non-Markovianity.

The $V(t)$ decay of a TLS results from two processes, the dephasing and the electronic population relaxation. In a pure dephasing case for which the electronic coupling vanishes, the populations remain constant and the volume is closely related to the decoherence function given by ${ }^{116,121,122} v(t)=$ $\exp (-\Lambda(t))$ with

$$
\Lambda(t)=\int_{0}^{\infty} d \omega J(\omega) \operatorname{coth}(\beta \omega / 2)(1-\cos (\omega t)) / \omega^{2} .
$$

The bumps in the non-monotonic decay of $v(t)$ or $V(t)$ occur with the same period as the correlation function $C(t)$ and depends only on the spectral density. The volume decays without bumps when $C(t)$ does not exhibit any oscillation, i.e. when the spectral density is very flat and broad. The population exchange when the electronic coupling is active, accelerates the decay of the volume and reduces or possibly flattens the bumps.

The volume evolution depends on the return of the collective mode back to its initial position leading to the bumps and on the non-adiabatic transition rate. Switching to the two-level-system one-coordinate representation, the volume behaves in a similar way as the autocorrelation function of the initial wave packet, which exhibits oscillations if the coordinate is not overdamped and has a decreasing norm due to the electronic transition. The decay of the initial wave packet norm depends on several factors, the time for reaching the crossing region and its usual Landau-Zener characteristics. The non-adiabatic dynamics is thus expected to be intrinsically non-Markovian when the collective mode is not overdamped (very thin spectral density) and the electronic decay is not too fast to avoid the blurring of the bumps.

In the CI case with a reference point where the electronic coupling vanishes, removing the off-diagonal coupling induced by the CM should lead to a pure dephasing case for the TM. A non-monotonic profile should be obtained as long as it is not overdamped. The population decay that flattens the bumps is due to the coupling bath. In the TLS-TC representation, it depends on the non-adiabatic evolution of a wave packet centered at the equilibrium position of the CM (since we consider here a symmetrical case).

\section{RESULTS}

We consider two diabatic excited states $|1\rangle$ and $|2\rangle$ and we assume that the system is initially prepared in the upper state $|2\rangle$ by an ultra fast excitation from a ground state $|0\rangle$ not included in the basis set. Modifying the parameters of the TLS model or of its spectral densities generates different shapes of the corresponding TLS-TC diabatic and adiabatic potential energy surfaces, in particular the CI position and the slopes at this point. This strongly influences the behavior of the electronic wave packet. The highly structured environment of the TLS is characterized by two peaked spectral densities $J_{t / c}(\omega)$ (Eq.(13)) with three main parameters (position, height, and width). Typical spectral densities used in the simulations are displayed in Fig.3a and the corresponding real part of the correlation functions (Eq.(12)) for $T=298 \mathrm{~K}$ are given in Fig. $3 \mathrm{~b}$.

The positions of the spectral density peaks fixed by the $\omega_{k}$ parameters (Eq.(13) nearly correspond to the effective frequencies of the TM and CM in the TLS-TC model when the functions are sharply peaked. We choose $\omega_{t}=1000 \mathrm{~cm}^{-1}$ and $\omega_{c}=500 \mathrm{~cm}^{-1}$ (different frequencies are considered in the supporting information $\left.{ }^{123}\right)$. The widths $\Gamma_{t / c}$ are proportional to the friction coefficient of the Ohmic spectral density of the residual bath in the TLS-TC model. In other words, this determines the damping of the TM or CM in this TLS-TC model. Two delta-function spectral densities ( with $\Gamma_{t / c}=0$ ) should lead to undamped TLS-TC dynamics, i.e., simple twodimensional dynamics. In most simulations, we take $\Gamma_{t}=\Gamma_{c}=$ $60 \mathrm{~cm}^{-1}$ but we will also illustrate the effect of a variation of 

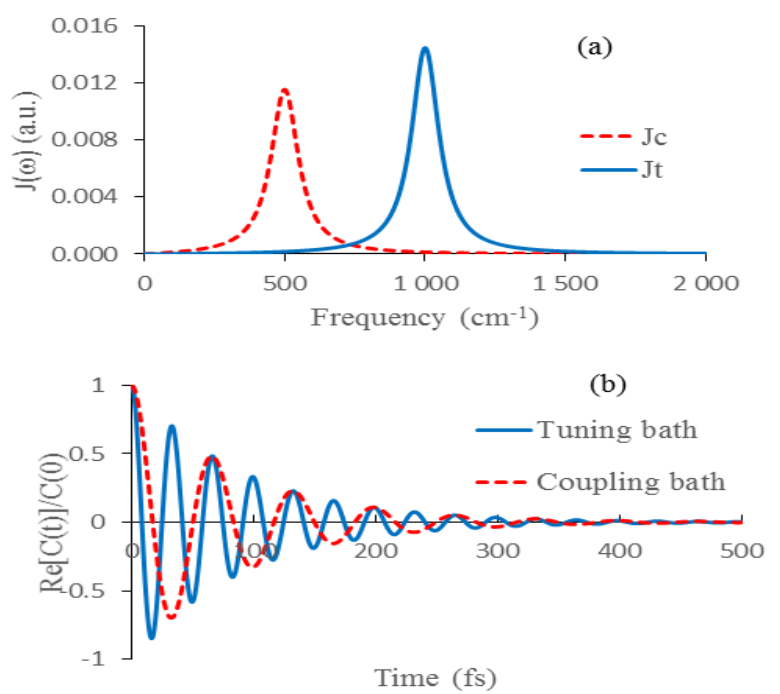

FIG. 3. (Color online) Panel (a): Spectral densities for the tuning (solid blue line) and coupling (dashed red line) baths of the TLS model. The renormalization energies (Eq.18) are $\lambda_{t}=188 \mathrm{~cm}^{-1}$ and $\lambda_{c}=300 \mathrm{~cm}^{-1}$, respectively. Panel (b): real parts of the corresponding normalized bath correlation functions $C_{t / c}(t)$ (Eq.(12)) for $T=298 \mathrm{~K}$. The parameters of the spectral densities (Eq.(13)) are $p_{t}=1.95 \times 10^{-11}$ a.u., $p_{c}=p_{t} / 2.5 ; \omega_{t}=4.5639 \times 10^{-3}$ a.u. $(1000$ $\left.\mathrm{cm}^{-1}\right), \omega_{c}=\omega_{t} / 2 ; \Gamma_{t}=\Gamma_{c}=2.7188 \times 10^{-4}$ a.u. $\left(60 \mathrm{~cm}^{-1}\right) . \lambda_{t}=$ $188 \mathrm{~cm}^{-1}$ and $\lambda_{c}=300 \mathrm{~cm}^{-1}$. The $p_{t / c}$ are taken as parameters to modify the renormalization energy $\lambda_{t / c}$ and therefore the linear couplings.

$\Gamma_{c}$. The weight $p_{t}$ (Eq.(13)) with constant $\Gamma_{t}$ determines the renormalization energy $\lambda_{t}$, which is added to the energy difference $\Delta$ to built $H_{S}$ of the TLS (Eq.(4)). In the TLS-TC model, modifying $p_{t}$ alters the distance $D_{t} / \Omega_{t}^{2}$ (Eq.(21)) between the minima of the diabatic parabolas. Varying $p_{c}$ modifies $\lambda_{c}$, which does not directly appear in the TLS system Hamiltonian but affects the linear coupling $D_{c}$ through the same relation (Eq.(21)) and therefore the shape of the adiabatic potential energy curves along the CM. In summary, frequencies, spatial displacement and slope of the electronic coupling depend on the spectral densities. The relative position in energy is fixed by the difference $\Delta$ between the minima corrected by $\lambda_{t}$. Finally, the reference point defining the initial equilibrium position of the bath determines the Franck-Condon (FC) region. We assume here that the equilibrium geometry of the ground electronic state also corresponds to that of the lower dark excited state $|1\rangle$ while the bright state $|2\rangle$ is displaced. When the reference point is located at the equilibrium position of the CM, the corresponding electronic coupling $W_{12}$ of the TLS model vanishes. By varying its position one could also explore situations where the electronic coupling $W_{12}$ is not zero in the center of the FC region.

We will present below different examples focusing on parameters which could be controlled by external laser fields (energy gap and initial state). We also illustrate the influence of the damping of the coupling mode, i.e., the impact of the residual friction coefficient $\eta_{c}$ (linked to the width via $\eta_{c}=2 \Gamma_{c}$ ) of the CM in the corresponding TLS-TC situation.
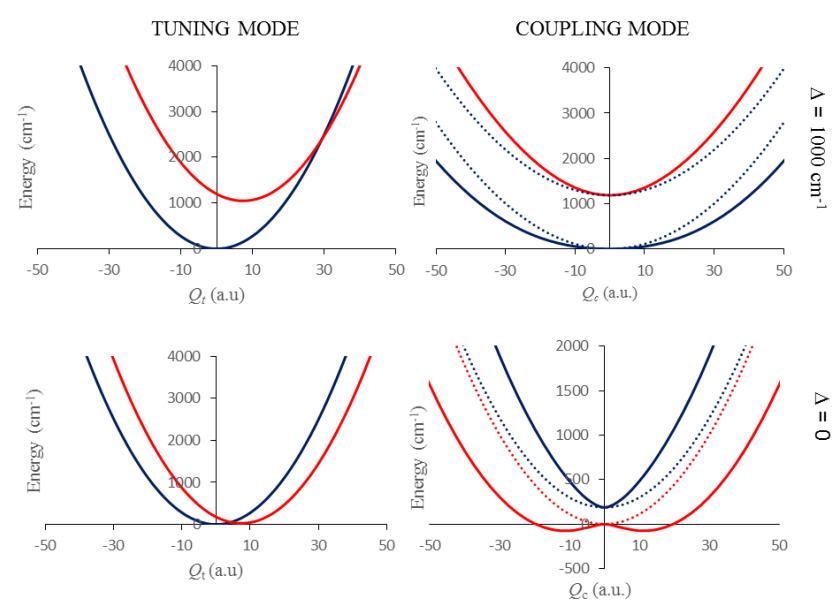

FIG. 4. (Color online) Potential energy curves of the TLS-TC model corresponding to the TLS with the spectral densities given in Fig.3 at the reference point $Q_{t}=0$ and $Q_{c}=0$. Top panels: diabatic energy gap $\Delta=1000 \mathrm{~cm}^{-1}$; lower panels: $\Delta=0$. Left panels: tuning mode $Q_{t}$ (the diabatic and adiabatic curves coincide). The curves correspond to an inverted Marcus-type crossing when $\Delta=1000 \mathrm{~cm}^{-1}$ and to a normal Marcus-type crossing when $\Delta=0$. Right panels: coupling mode $Q_{c}$, dashed lines diabatic curves, solid lines adiabatic curves. The renormalization energy $\lambda_{t}=188 \mathrm{~cm}^{-1}$ is added to $\varepsilon_{2}$. $\lambda_{c}=300 \mathrm{~cm}^{-1}$.

The supporting information analyzes the variation of other molecule-dependent parameters (linear couplings and effective frequencies) $)^{123}$.

In the HEOM simulations, the correlation function of each bath is expanded on three exponential functions. Two sets of coefficients in Eqs.(12) and (17) come from the poles of the chosen spectral density (Eq.(13)). A single Matsubara term is enough to ensure convergence at room temperature. A high hierarchy level is necessary for analyzing the statistical distribution even if the populations converge at a lower level. Convergence of the expansions (Eqs.(25) and (29)) may require about 20 or 25 terms and therefore the same hierarchy level. A typical computation at levels 20/25 involves 230230/736281 matrices respectively. The integration is carried out by the CashKarp Runge-Kutta algorithm with adaptative time step ${ }^{124}$. The wave packet is computed on a two-dimensional grid by the non-adiabatic split-operator algorithm ${ }^{125}$ using only the system Hamiltonian of the TLS-TC model $\tilde{H}_{S}$. For each dimension, the grid contains $2^{10}$ points. The time step is 10 a.u.

\section{A. Variation of the energy gap}

Fig.4 presents cuts in the TLS-TC potential energy surfaces along the TM (left panel) and CM (right panel) at the reference position $\left(Q_{t}=0\right.$ and $\left.Q_{c}=0\right)$ for two typical situations in which the adiabatic lower curve along the CM has either a single minimum or a barrier at the equilibrium position. 


\section{Environmental statistical distribution}

Fig.5 gives iso-value contours of the projected distribution $P_{\alpha}\left(x_{t / c}, t\right)$ (Eq. (25)) on the collective bath coordinate in each electronic state for the two contrasting cases illustrated in Fig.4. In each case, the initial distribution is a Gaussian one in the upper excited state. When the electronic gap is $\Delta=1000 \mathrm{~cm}^{-1}$ (upper panels of Fig.4), the environmental dynamics of the TM presented in the left panels of Fig.5 are similar to the behavior already obtained in a previous work in a one-dimensional model ${ }^{112}$. One observes the initial displacement of the average position towards the equilibrium position of the upper state after the preparation in the FC region and during about 50fs the average position is similar to the trajectory of a Gaussian wave packet with a decreasing norm due to the non-adiabatic transition induced by the CM. This can be related to the so-called "Zeno time" as analyzed in ref. ${ }^{73}$. The component appearing in the lower state also exhibits damped oscillations. The equilibrium distribution in the final state is reached after $250 \mathrm{fs}$ in agreement with the correlation time scale (Fig.3b). For the coupling mode (right panels), the Gaussian distribution smoothly melts at the initial equilibrium position. The density appears in the lower state with two symmetric lobes, fulfilling for a short time (about 10fs) the expected node at the equilibrium position since the electronic coupling is zero at this point. The damping then evolves towards a uni-modal distribution in agreement with the shape of the potential energy curves with a single minimum.

The distribution $P_{\alpha}\left(x_{t}, t\right)$ (Eq.(25)) of the tuning bath in the $\Delta=0$ case (lower panels of Fig. 4 ) evolves during the first fifty femtoseconds as in the previous example but the final norm is not zero since the final population is the same in both states. The asymptotic distribution is Gaussian in both states and centered at the respective equilibrium positions. When the energy gap decreases, the profile of the diabatic and adiabatic lower potential energy curves along the coupling mode are very different, with either a minimum or a barrier at the equilibrium position. Interestingly, the asymptotic distribution of the coupling bath becomes bi-modal. Even if for the reduced electronic system Hamiltonian $H_{S}$ the diabatic and adiabatic basis sets coincide for the chosen reference point, the full entangled system-bath dynamics provide a vibrational asymptotic distribution that correctly "feels" the adiabatic potential of the coordinate representation. This reveals the efficiency of the information extracted from the auxiliary matrices.

Fig.6 compares the full statistical distribution $P_{\alpha}\left(x_{t}, x_{c}, t\right)$ (Eq.29) in the two diabatic electronic states at two times with the square modulus of the two-dimensional undamped wave packet $\left|\Psi_{\alpha}\left(Q_{t}, Q_{c}, t\right)\right|^{2}$ in the diabatic representation. We illustrate only the case with $\Delta=1000 \mathrm{~cm}^{-1}$ (first two rows in Fig. 5 and corresponding TLS-TC model in the first row of Fig.4). The first chosen time $t=25 \mathrm{fs}$ is shorter than the Zeno time so that the behaviors are expected to be very close. The only noticeable difference concerns the nodal line in the wave packet map that is already blurred at this short time in the damped case. On the contrary, for $t=120 \mathrm{fs}$, the damped distribution has nearly reached its asymptotic shape around the equilibrium position of the lower electronic state while the undamped wave packet remains fully delocalized.

For the same example with $\Delta=1000 \mathrm{~cm}^{-1}$, Fig.7 gives the imaginary part of the coherence (Eq.(30)) in the diabatic representation for the damped and the undamped wave packet. At short time $t=25 \mathrm{fs}$, this confirms the efficiency of the extraction of information from the off diagonal elements $M_{n, m}^{\alpha \beta}(t)$ (Eq.(28)). At longer time $t=120 \mathrm{fs}$, the coherence becomes two orders of magnitude smaller in the damped case while it remains at the same order of magnitude in the undamped case, as expected.

We compare in Fig.8 the two-dimensional distribution in the lower excited state in the diabatic (left panels) and in the adiabatic (right panels) representations, for the two gaps $\Delta=$ $1000 \mathrm{~cm}^{-1}$ and $\Delta=0$ illustrated in Fig. 4 and for two times, a short ( $t=25 \mathrm{fs}$ ) and a long one ( $t=240 \mathrm{fs}$ ). The adiabatic representation is obtained through Eqs.(32) using the parameters of the TLS-TC model to build the diabatic-to-adiabatic transformation matrix at every point of the grid.

\section{Electronic dynamics}

Fig.9 gives the population of the TLS model in the upper excited state when the energy gap $\Delta$ decreases from 1500 $\mathrm{cm}^{-1}$ to zero in the situation where the spectral densities are centered at $\Omega_{c}=500 \mathrm{~cm}^{-1}$ for $J_{c}(\omega)$ and $\Omega_{t}=1000 \mathrm{~cm}^{-1}$ for $J_{t}(\omega)$ with renormalization energies $\lambda_{c}=150 \mathrm{~cm}^{-1}$ and $\lambda_{t}=$ $188 \mathrm{~cm}^{-1}$. The population evolution for the undamped TLSTC is shown in Fig.9b. One observes a perfect agreement during the first thirty femtoseconds before the damping operates. This corresponds to a period of the tuning mode as may be seen in Fig.9c where is drawn the first moment of the collective mode $X_{t} / D_{t}$ of the tuning bath in the upper electronic state. The damped TLS decay curves show markedly different profiles from a smooth overdamped decay (long dashes in Fig.9) for the large gap $\Delta=1500 \mathrm{~cm}^{-1}$ to damped oscillatory decay with variable periods of partial recurrence and asymptotic values. The different recurrence periods and initial decay yields during the first oscillation are already observed in the undamped TLS model (Fig.9b).

These general trends may be explained by the way the FC wave packet will explore the CI region. Varying the diabatic gap modifies the location of the CI and the slopes at the crossing. In the present examples, the CI is of the sloped type (see upper panels in Fig.4), except for the case $\Delta=0$ leading to a peaked case (see lower panels in Fig. 4$)^{30}$. For the largest gap $\Delta=1500 \mathrm{~cm}^{-1}$, the $\mathrm{CI}$ is above the $\mathrm{FC}$ region and the smooth decay occurs via tunneling. In the case $\Delta=500 \mathrm{~cm}^{-1}$ on the contrary, the $\mathrm{CI}$ is close to the turning point. In the $\Delta$ $=0$ case, the $\mathrm{CI}$ is very close to the FC region, thus leading to early Stueckelberg oscillations ${ }^{126}$.

Another way to explain the different oscillation timescales in the population evolution is the way the initial wave packet is expanded in the eigen vibronic basis set. This superposition depends on the gap $\Delta$ and involves different energy gaps between the main eigenstates and therefore different oscillation periods. The variation of $\Delta$ also leads to different asymptotic populations (Fig.9a) and different average positions of 

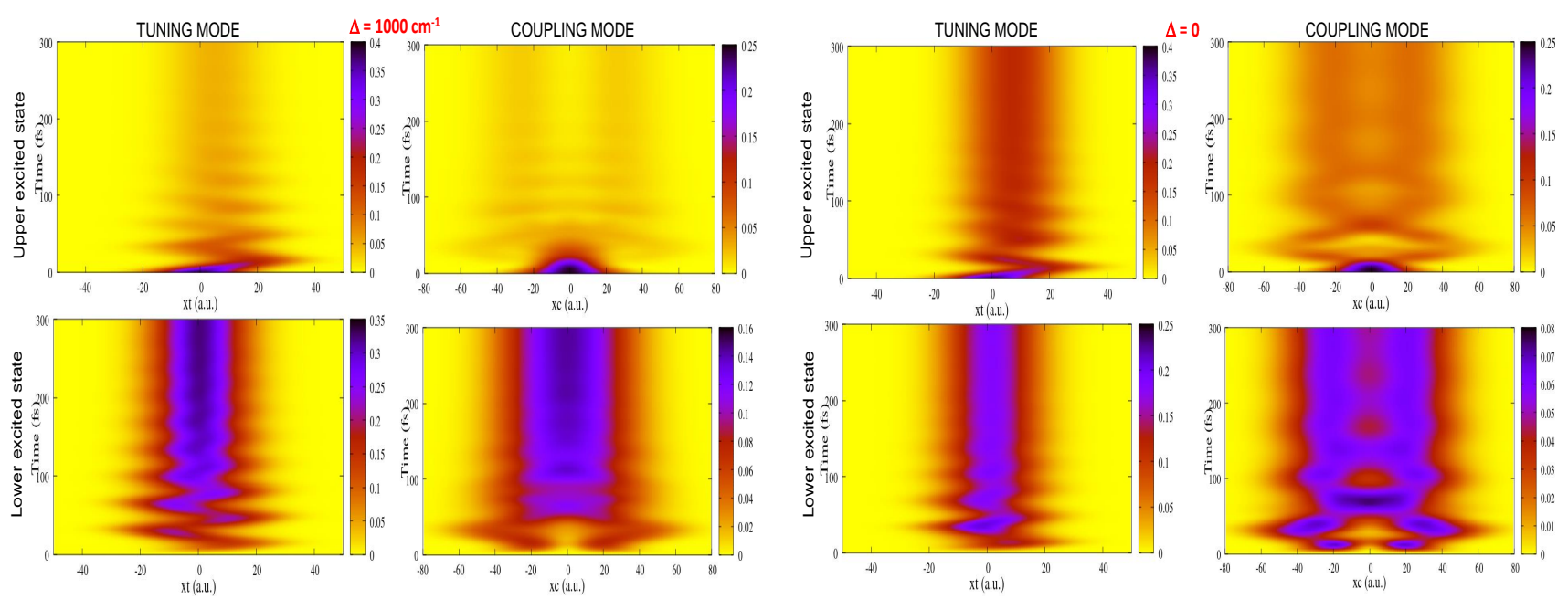

FIG. 5. Projected statistical distributions $P_{\alpha}\left(x_{t / c}, t\right)$ (Eq.25) on the collective coordinates computed in the TLS model for the corresponding TLS-TC model represented in Fig.4. The color code gives $P\left(x_{t / c}, t\right) \times 10$. The left/right panels show the tuning/coupling baths, respectively. The diabatic energy gaps are $\Delta=1000 \mathrm{~cm}^{-1}$ and $\Delta=0 . \lambda_{t}=188 \mathrm{~cm}^{-1}$ and $\lambda_{c}=300 \mathrm{~cm}^{-1}$. The spectral densities are given in Fig.3a. $T=$ 298 K.
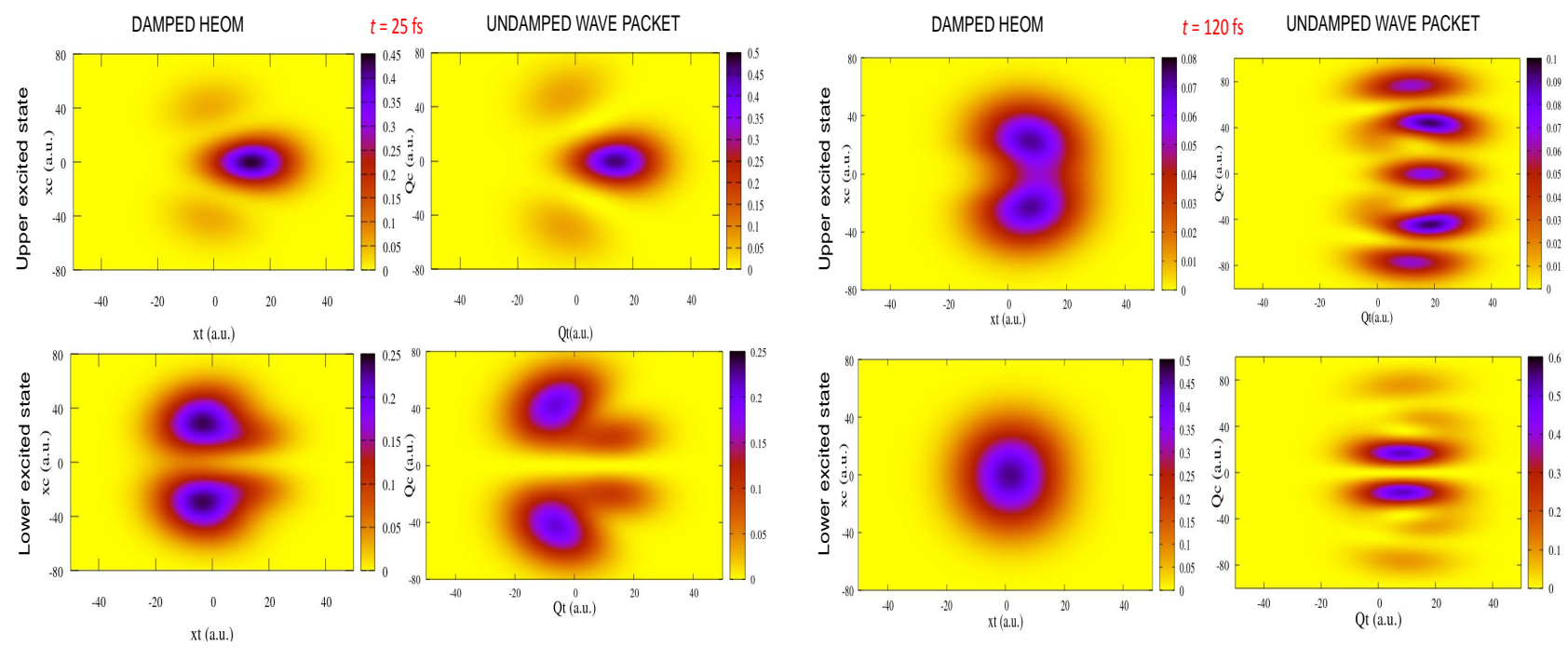

FIG. 6. Statistical distributions $P_{\alpha}\left(x_{t}, x_{c}, t\right)$ (Eq.(29)) computed by HEOM in the TLS model and undamped wave packet $\left|\Psi\left(Q_{t}, Q_{c}, t\right)\right|^{2}$ of the TLS-TC model represented in the upper panels of Fig.4, for two times, $t=25 \mathrm{fs}$ and $t=120 \mathrm{fs}$. The color code gives $\left(x_{t}, x_{c}, t\right) \times 10^{3}$. The gap between the two diabatic minima is, $\Delta=1000 \mathrm{~cm}^{-1}, \lambda_{t}=188 \mathrm{~cm}^{-1}$ and $\lambda_{c}=300 \mathrm{~cm}^{-1}$. The spectral densities are given in Fig. $3 \mathrm{a}$. $T=298 \mathrm{~K}$.

the collective mode (Fig.9c). The final equilibrium Botzmann mixture concerns the eigen vibronic states and not only the electronic states ${ }^{66}$.

Finally Fig.9d gives the volume of the accessible states in the Bloch sphere, which is a signature of non-Markovianity when the decay is not monotonic. The non-Markovianity signature is given rigorously by the integrals of the region where the volume increases but the shape of the bumps provides a qualitative information. In the context of non-adiabatic dynamics, we have already discussed ${ }^{112}$ that this volume is closely linked to the correlation function of the wave packet in the corresponding effective mode model. It reveals the tendency of both the electronic and vibrational wave packets to return towards the initial position. The black solid curve in Fig.9d gives the volume for the pure dephasing case of the only TM. The bumps merely correspond to the recurrences of the bath correlation function of the TM alone $C(t)$ (see Fig.3b) and should match with the oscillations of the wave packet in the effective coordinate model. When the CM is taken into account, the electronic transition (see populations in Fig.9b) increases the decay rate and blurs the bumps. Electronic dynamics of a CI described by a TLS is non Markovian in the present example even if the population decay seems smooth (however non exponential) as it is the case for $\Delta=1500 \mathrm{~cm}^{-1}$ (long dashes). 

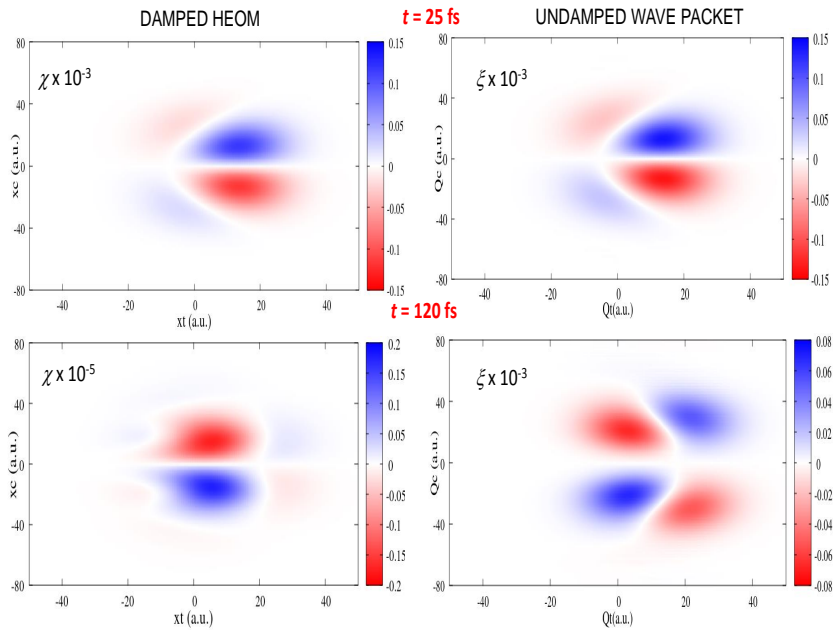

FIG. 7. Electronic coherence $\chi\left(x_{t}, x_{c}, t\right)$ (Eq.(30)) computed by HEOM in the TLS model and wave packet coherence $\xi\left(Q_{t}, Q_{c}, t\right)$ for two times, $t=25 \mathrm{fs}$ and $t=120 \mathrm{fs}$. The corresponding TLS-TC model is represented in the upper panels of Fig.4. The gap between the two diabatic minima is $\Delta=1000^{-1}, \lambda_{t}=188 \mathrm{~cm}^{-1}$ and $\lambda_{c}=300$ $\mathrm{cm}^{-1} . T=298 \mathrm{~K}$.

\section{B. Initial electronic state}

We now examine the difference of behaviors related to the initial preparation of the electronic system. The energy gap is $\Delta=1000 \mathrm{~cm}^{-1}$ and the renormalization energies are $\lambda_{t}=$ $188 \mathrm{~cm}^{-1}$ and $\lambda_{c}=150 \mathrm{~cm}^{-1}$. Control strategies with ultrafast designed laser fields could modify the initial superposed electronic state. For the examples in the previous section, the initial state is prepared as $|2\rangle$. As the center of the $\mathrm{FC}$ region is located at $Q_{c}=0$, the electronic coupling vanishes and the diabatic and adiabatic electronic states coincide. The initial state is thus a pure state, which does not involve electronic coherence. The relaxation of the coupling vibrational bath and the evolution towards equilibrium in the final state maintain the symmetry as it has been illustrated in the previous examples.

When the initial state is a superposed state, electronic coherence is created and persists during the timescale of the correlation function (about $200 \mathrm{fs}$ ). Panels (a) and (b) of Fig.10 give two examples for the initial superposed states $\left|\chi_{1}\right\rangle=(|1\rangle+\sqrt{9}|2\rangle) / \sqrt{10}$, and $\left|\chi_{2}\right\rangle=(|1\rangle+|2\rangle) / \sqrt{2}$, respectively. In addition to the initial electronic coherence, the new behavior is the breaking of symmetry in the vibrational relaxation and evolution towards equilibrium of the coupling bath. This is illustrated for $P\left(x_{c}, t\right)$ in state $|1\rangle$. This unexpected vibrational effect is already observed in the evolution of the undamped two-dimensional wave packet as can be seen in panel (d) of Fig.10 where we compare the average position of CM computed by the undamped two-dimensional wave packet and by HEOM. The curves are labeled UNDAMPED1 and DAMPED1 for $\left|\chi_{1}\right\rangle$, and UNDAMPED2, DAMPED2 for $\left|\chi_{2}\right\rangle$. The dissymmetry comes from the interferences of the left or right parts of the upper wave packet when it makes a transition due to the antisymmetrical electronic coupling. The initial wave packet in the lower state has everywhere the same sign while the decaying parts get the opposite sign due to the coupling. The resulting wave packet in the lower state becomes asymmetrical. This effect is illustrated in panel (c) of Fig.10 where we give the square modulus of the undamped wave packet in the lower state at a short time $t=12 \mathrm{fs}$.

\section{Variation of $\Gamma_{c}$}

Fig.11 compares iso-contours of the projection $P\left(Q_{t}, t\right)$ (Eq.(24)) in the lower electronic state $|1\rangle$ of the localization density probability for the undamped two-dimensional wave packet dynamics with the statistical distribution $P\left(x_{c}, t\right)$ (Eq.25) for increasing width of $J_{c}(\omega)$, i.e., increasing the friction coefficient of the residual bath in the TLS-TC model. The energy gap is $\Delta$ is $1000 \mathrm{~cm}^{-1}$ and the two renormalization energies are $\lambda_{t}=188 \mathrm{~cm}^{-1}$ and $\lambda_{c}=150 \mathrm{~cm}^{-1}$. The adiabatic lower potential energy curve has a single minimum along the CM. When the width $\Gamma_{c}$ of the spectral density $J_{c}(\omega)$ is modified, the weight $p_{c}$ is adapted to impose the same $\lambda_{c}$ and therefore the same linear coupling $D_{c}$. The damping begins after $30 \mathrm{fs}$ and one sees the blurring of the characteristic nodal line of the distribution and the evolution towards the final distribution is faster with increasing $\Gamma_{c}$.

\section{CONCLUSIONS}

The main result of this work is to illustrate the ability of the powerful tool provided by HEOM to get an interesting information about the statistical distribution of the position of the collective mode associated with different baths from the auxiliary matrices. We have extended for two uncorrelated baths the methodology suggested by Shi et al. ${ }^{76,77}$ by deciphering the partial level related to each bath for each auxiliary matrix. We have derived the projected and the twodimensional distributions of the tuning and coupling modes of a conical intersection from the minimal TLS electronic partitioning. This strategy already proposed in the literature ${ }^{74,75}$ could provide a better computational scaling than the more familiar two-state two-coordinate representation ${ }^{72}$. The present work shows how to complement this approach by visualizing the evolution of the environmental distributions in each electronic state that correspond to the square modulus of the dissipative wave packets. When the electronic dynamics induces a strong restructuring of the environment, one may visualize the departure from the initial Gaussian equilibrium distribution (within the initial factorization assumption). By using the parameters of the associated TLS-TC model, we have also illustrated how to derive the adiabatic distributions from those computed in the diabatic representation in the TLS system. In the present work, we have considered only a very symmetrical model and an initial reference point located in a region where the electronic coupling vanishes so that diabatic and adiabatic states coincide at this point. As a consequence, the minimal electronic system will not give any information on the pro- 

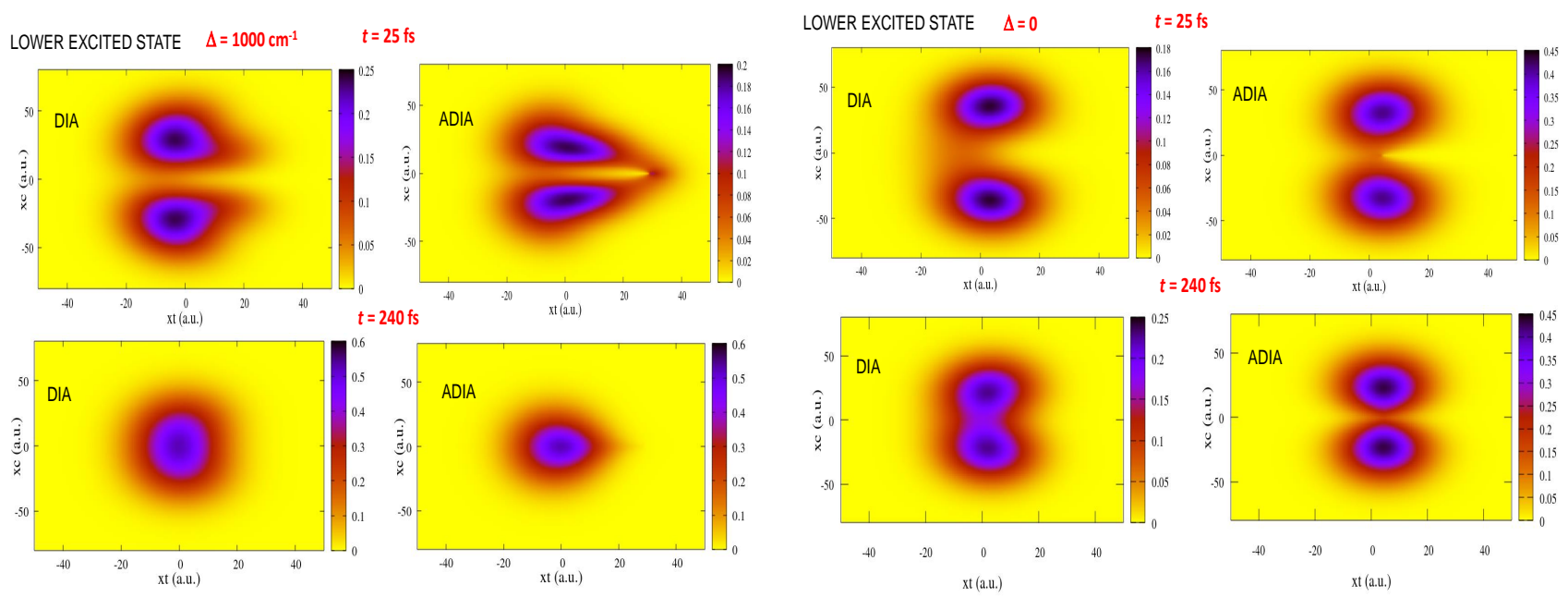

FIG. 8. Statistical distributions in the lower excited state $P_{1}^{\text {dia }}\left(x_{t}, x_{c}\right)$ (Eq.(29)) and $P_{1}^{a d i a}\left(x_{t}, x_{c}\right)$ (Eq.(32)) computed by HEOM in the TLS model and using the parameters of the TLS-TC model to obtain the adiabatic map. The gaps are $\Delta=1000 \mathrm{~cm}^{-1}$ and $\Delta=0$ (see Fig.4). The distributions are given for two times, $t=25 \mathrm{fs}$ and $t=240 \mathrm{fs}$. The color code gives $P\left(x_{t}, x_{c}\right) \times 10^{3} . \lambda_{t}=188 \mathrm{~cm}^{-1} \mathrm{and}_{c}=300 \mathrm{~cm}-1 . T=$ 298K.
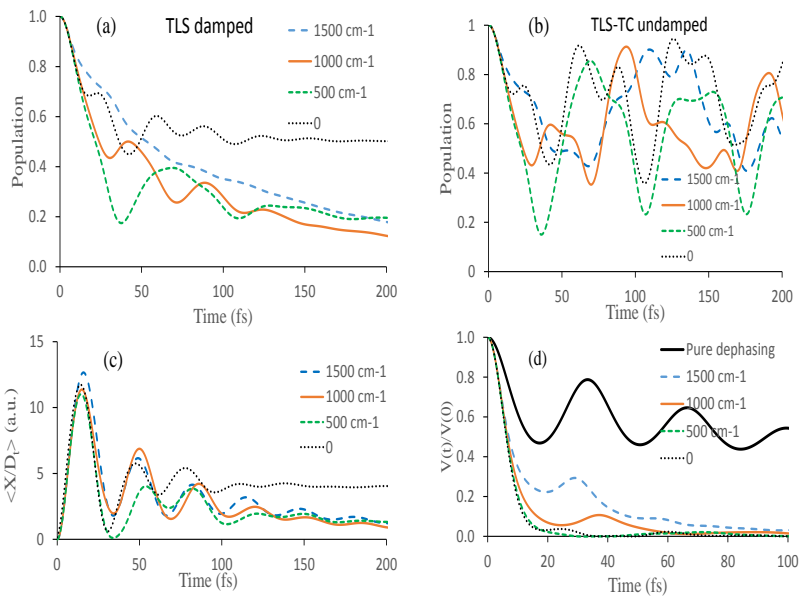

FIG. 9. (Color online) Variation of dynamical quantities with the electronic energy gap between the diabatic minima $\Delta$. Upper panels : Population in the initial upper electronic state; panel (a)TLS damped HEOM dynamics, panel (b) undamped TLS-TC wave packet dynamics (b). Panel (c) : first moment of the collective TM in the upper electronic state extracted from the auxiliary matrices. Panel (d) Volume of the accessible states in the Bloch sphere. The parameters of the spectral densities $J_{t}(\omega)$ and $J_{c}(\omega)$ are $\Omega_{t}=1000 \mathrm{~cm}^{-1}, \lambda_{t}=$ $188 \mathrm{~cm}^{-1}$ and $\Omega_{c}=500 \mathrm{~cm}^{-1}, \lambda_{c}=150 \mathrm{~cm}^{-1}, T=298 \mathrm{~K}$.

jected electronic coherence if the initial state is a single state. However, maps of the real and imaginary parts of the coherence corresponding to the cross product of the wave packet components in the two electronic states may be nicely derived from the auxiliary matrices.

Working in the TLS model with HEOM is the opposite strategy to the projection of electronic dynamics from a full dimension wave packet and it is particularly rewarding to see
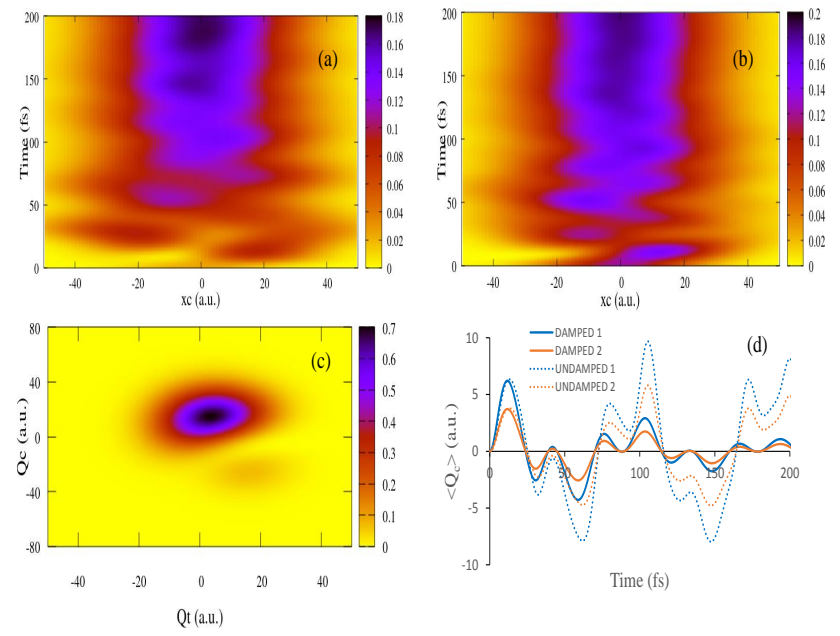

FIG. 10. (Color online) Statistical distribution $P\left(x_{c}, t\right)$ projected on the coupling mode in the lower excited state $|1\rangle$ for two initial superposed states. Panels (a) and (b): Preparation in states $\left|\chi_{1}\right\rangle=(|1\rangle+\sqrt{9}|2\rangle) / \sqrt{10}$ and $\left|\chi_{2}\right\rangle=(|1\rangle+|2\rangle) / \sqrt{2}$, respectively (The color code gives $P\left(x_{c}\right) \times 10$ ); panel (c) distribution of the undamped wave packet in the lower excited state at time $t=12 \mathrm{fs}$ for state $\left|\chi_{2}\right\rangle$ (the color code gives $|\Psi|^{2} \times 10^{3}$; panel (d): Average position of the effective mode $Q_{c}(t)$ computed with the undamped twodimensional wave packet and of the collective coordinate $x_{c}(t)$ obtained from the damped HEOM. $\lambda_{t}=188 \mathrm{~cm}^{-1}$ and $\lambda_{c}=150 \mathrm{~cm}^{-1}$, $\Delta=1000 \mathrm{~cm}^{-1}, T=298 \mathrm{~K}$.

the efficiency of this HEOM technique. However, high levels of hierarchy are required when the distribution becomes bi- or multi-modal since the distribution is expanded on a Hermite polynomial basis set and convergence may require higher levels than the simple population computation. We have used a home made code parallelized with OpenMP. However other 

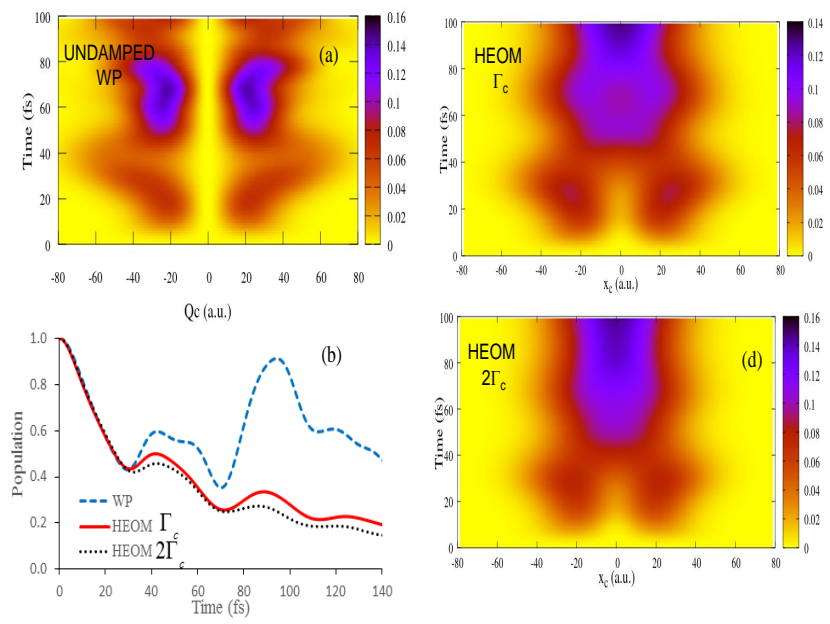

FIG. 11. (Color online) Comparison of the undamped twodimensional wave packet projected dynamics $P\left(Q_{c}, t\right)$ (panel (a)) and the projected statistical distribution for the CM $P\left(x_{c}, t\right)$ in the lower electronic state for increasing width $\Gamma_{c}$ and the same linear coupling $D_{c}$ (panels (c) and (d)). The color code gives $P \times 10$. Panel (b) gives the decay of the population for the undamped wave packet and for the HEOM dynamics with increasing width. $T=298 \mathrm{~K}, \Delta=1000$ $\mathrm{cm}^{-1}, \lambda_{t}=188 \mathrm{~cm}^{-1}$ and $p_{c}$ is adjusted to give the same $\lambda_{c}=150$ $\mathrm{cm}^{-1}$ when $\Gamma$ is modified.

implementations on new architectures such as GPU could be useful $^{127}$.

The inclusion of the TM and CM in their respective bath are expected to generate two highly structured spectral densities and therefore long correlation times and strongly nonMarkovian dynamics as confirmed by the non monotonic decay of the volume of accessible states in the Bloch sphere in our example. As already discussed in a previous work ${ }^{112}$, the volume evolution is closely linked to the autocorrelation function of the nonadiabatic wave packet of the corresponding TLS-TC model. The back flow of the electronic energy is induced by the return of the collective mode towards its initial position. Another measure of non-Markovianity during exciton-phonon dynamics has also been extracted from the auxiliary matrices ${ }^{128}$.

It is worthwhile noting that even if we have treated the specific example of a conical intersection, the method by itself has a large panel of applications to analyze the electronvibration dynamics of complex systems such as excitonphonon dynamics. HEOM offers the advantage of easily accounting for high temperature and this paves the way towards interesting applications for instance by considering baths at different temperatures ${ }^{68,129,130}$. Moreover, the possibility to include radiative couplings and exploit laser induced conical intersection as recently suggested ${ }^{131,132}$ in order to control the electronic dynamics is also a possible future application. Optimal control with laser fields has recently been implemented with HEOM $^{133}$ and the new tool could provide an interesting information about the controlled environmental dynamics.

\section{SUPPLEMENTARY MATERIAL}

The supplemental material presents some complementary examples of electronic dynamics by varying the linear couplings $D_{t / c}$ of the TLS-TC model extracted from the TLS model (Eq.(19)) or the peak positions of the spectral densities fixed by the parameters $\omega_{t / c}$ (Eq.(13)).

\section{ACKNOWLEDGMENTS}

We warmly acknowledge Dr A.X. Chin for interesting discussions. One of us (EM) acknowledges support from the ANR-DFG COQS, under Grant No. ANR-15-CE30-0023-01. This work has been performed within the French GDR 3575 THEMS.

\section{Appendix A: HEOM for two uncorrelated baths}

Solving the hierarchical equations of motion for a conical intersection requires a set of equations for two baths. In this derivation, we mainly assume that they are uncorrelated. Such expressions have been derived in several works, for particular spectral densities ${ }^{74,75}$. Here, we essentially aim at recalling the derivation of the coupled equations and the assumptions that we used.

\section{Auxiliary matrices in the two-uncorrelated-bath case}

The reduced density matrix $\rho_{S, I}(t)$ for the system in interaction representation $\rho_{S, I}(t)=e^{i H_{0} t} \rho_{S}(t) e^{-i H_{0} t}$ with $H_{0}=$ $H_{S}+H_{B}$, , is given by the partial trace over the bath $B$ of the time evolution of the total density matrix,

$$
\rho_{S, I}(t)=\operatorname{Tr}_{B}\left[T^{(+)} e^{\int^{t} d \tau L(\tau)} \rho_{B}^{e q} \rho_{S, I}(0)\right]
$$

where $T^{(+)}$is a time-ordering operator and a factorization is assumed at the initial time $t=0 . L(t)$ is the Liouvillian in interaction representation, which reads

$$
L(t) .=-\frac{i}{\hbar^{2}}\left[S_{t}(t) \cdot B_{t}(t)+S_{c}(t) \cdot B_{c}(t), .\right]=L_{t}(t) \cdot+L_{c}(t) .
$$

The partial trace over $B$ is a successive trace over bath $B_{t}$ and bath $B_{v}: \operatorname{Tr}_{B}[\cdot]=\operatorname{Tr}_{B_{t}}\left[\operatorname{Tr}_{B_{c}}[\cdot]\right]$ and $\rho_{B}^{e q}=\rho_{B_{t}}^{e q} \rho_{B_{c}}^{e q}$.

In the case of harmonic oscillators with linear coupling, it has been shown that the second-order cumulant expansion of Eq. (A1) is exact ${ }^{93,94,101,134}$,

$$
\rho_{S, I}(t)=T^{(+)} e^{\int^{t} d \tau \int_{0}^{\tau} d t^{\prime} T r_{B}\left[L(\tau) L\left(t^{\prime}\right) \rho_{B}^{e q}\right]} \rho_{S, I}(0)
$$

Owing to this feature, $\int_{0}^{\tau} d t^{\prime} \operatorname{Tr}_{B}\left[L(\tau) L\left(t^{\prime}\right) \rho_{B}^{e q}\right]$ in Eq.(A3) is a second-order memory kernel similar to the one found in 
second-order perturbation theory. It will be denoted $\mathscr{K}(\tau)=$ $\int^{\tau} d t^{\prime} K^{(2)}\left(\tau, t^{\prime}\right)$. First, one can easily derive from Eq.(A2) an expression for the two-bath case,

$$
\begin{array}{r}
K^{(2)}\left(\tau, t^{\prime}\right) \cdot=\operatorname{Tr}_{B}\left[L(t) L(\tau) \cdot \rho_{B}^{e q}\right] \\
=\operatorname{Tr}_{B_{t}}\left[L_{t}(\tau) L_{t}\left(t^{\prime}\right) \cdot \rho_{B_{t}}^{e q}\right]+\operatorname{Tr}_{B_{c}}\left[L_{c}(\tau) L_{c}\left(t^{\prime}\right) \cdot \rho_{B_{c}}^{e q}\right] \\
+\operatorname{Tr}_{B}\left[L_{t}(\tau) L_{c}\left(t^{\prime}\right) \cdot \rho_{B}^{e q}\right]+\operatorname{Tr}_{B}\left[L_{c}(\tau) L_{t}\left(t^{\prime}\right) \cdot \rho_{B}^{e q}\right] .
\end{array}
$$

When the two baths are not correlated, cross-terms in Eq.(A4) are considered as negligible and discarded for the rest of the present derivation. For Eq.(A4), one can apply strategies similar to the one used for second-order perturbation theory. By using usual open quantum-system methods ${ }^{135}$, one can eliminate the bath and cast its whole influence in two-time correlation functions $C_{t / c}(t-\tau)=\operatorname{Tr}_{B_{t / c}}\left[B_{t / c}(t) B_{t / c}(\tau) \rho_{B_{t / c}}^{e q}\right]$ for the tuning and coupling mode respectively:

$$
\begin{array}{r}
\int_{0}^{\tau} d t^{\prime} K^{(2)}\left(\tau, t^{\prime}\right) . \\
=-\sum_{j=t / c}\left[S_{j}(\tau), \int_{0}^{\tau} d t^{\prime} C_{j}\left(\tau-t^{\prime}\right) S_{j}\left(t^{\prime}\right) \cdot-\{h . c .\}\right] .
\end{array}
$$

In this respect, using correlation function parametrizations $C_{t / c}\left(\tau-t^{\prime}\right)$ given in Eq.(16) and its complex conjugate $C_{t / c}^{*}\left(\tau-t^{\prime}\right)$ (Eq.(17)) and following strategies proposed for instance in ${ }^{92}$ and $^{94,101,134}$, equation (A5) is separable into a sum of operators $\mathscr{K}_{k=\left\{j, l_{j}\right\}}$,

$$
\int_{0}^{\tau} d t^{\prime} K^{(2)}\left(\tau, t^{\prime}\right) \cdot=\mathscr{K}(\tau) \cdot=\sum_{j=1}^{2} \sum_{l_{j}=1}^{n_{\text {cor }, j}} \mathscr{K}_{k=\left\{j, l_{j}\right\}}(\tau) .
$$

where $\mathscr{K}_{k=\left\{j, l_{j}\right\}}(\tau)$ is assigned to each exponential element $l_{j}$ of the correlation function of bath $j=\{t, c\}$. In addition, we decompose it as

$$
\mathscr{K}_{k=\left\{j, l_{j}\right\}}(\tau)=\Phi_{j}(\tau) W_{k}(\tau)
$$

where we have defined

$$
\Phi_{j}(\tau) \cdot=-\left[S_{j}(\tau), \cdot\right]
$$

and

$$
W_{k}(\tau)=\int_{0}^{\tau} d t^{\prime} e^{i \gamma_{k}\left(\tau-t^{\prime}\right)} \Theta_{k}\left(t^{\prime}\right)
$$

with

$$
\Theta_{k}\left(t^{\prime}\right) \cdot=\alpha_{j, l_{j}} S_{j}\left(t^{\prime}\right) \cdot-\tilde{\alpha}_{j, l_{j}} \cdot S_{j}\left(t^{\prime}\right) .
$$

By Fourier-Laplace transforming Eq.(A1) ${ }^{48,136}$, one can recast Eq. (A3) as

$$
\rho_{S, I}[\omega]=\int_{0}^{+\infty} d t e^{-i \omega t} \rho_{S, I}(t)=\int_{0}^{+\infty} d t e^{-i \omega t+\int_{0}^{t} d \tau \mathscr{K}(\tau)} \rho_{S, I}(0) .
$$

Finally, we define auxiliary matrices ${ }^{101,136}$ as

$\rho_{n_{1}, \cdots, n_{K}}^{(v)}[\omega]=\int_{0}^{+\infty} d t \prod_{k=1}^{K} W_{k}^{n_{k}}(t) e^{-i \omega t+\int_{0}^{t} d \tau \mathscr{K}(\tau)} \rho_{S, I}(0)$

It is interesting to note that $\rho_{S, I}=\rho_{0, \cdots, 0}^{(0)}$ is the system density matrix we are looking for. To better clarify the notations, let us emphasize that

- $k=\left\{l_{j}, j\right\}$ is a global index related to an exponential element $l_{j}$ of the $j$ 's bath correlation function and the $j=\{t, c\}$ bath itself.

- $n_{k}$ is an integer that is a power of $W_{k}$.

- $K=n_{c o r, 1}+n_{c o r, 2}$ is the total number of exponential functions in the correlation function parameterization (Eq.16),

- $\mathbf{n}=\left\{n_{1,1}, \cdots, n_{n_{c o r}, 1}, n_{1,2}, \cdots, n_{n_{c o r}, 2}\right\}$ is an index vector bound to a hierarchy matrix.

- $(v)$ is the hierarchy level of the matrix defined such that $v=\sum_{i=1}^{K} \mathbf{n}_{i}$

\section{Derivation of the hierarchical equations of motions}

For any $k=\left\{l_{j}, j\right\}$, one has the following relation, 


$$
\begin{aligned}
& -i \omega \rho_{n_{1}, \cdots, n_{K}}^{(v)}[\omega]+\sum_{k=1}^{K} \Phi_{j}(t) \rho_{n_{1}, \cdots, n_{k}+1, \cdots, n_{K}}^{(v+1)}[\omega]= \\
& \int_{0}^{+\infty} d t \prod_{k=1}^{K} W_{k}^{n_{k}}(t)\left(-i \omega+\sum_{k=1}^{K} \Phi_{j}(t) W_{k}(t)\right) e^{-i \omega t+\int_{0}^{t} d \tau \mathscr{K}(\tau)} \rho_{S, I}(0) .
\end{aligned}
$$

By performing an integration by parts, one obtains

$$
\begin{aligned}
& -i \omega \rho_{n_{1}, \cdots, n_{K}}^{(v)}[\omega]+\sum_{k=1}^{K} \Phi_{j}(t) \rho_{n_{1}, \cdots, n_{k}+1, \cdots, n_{K}}^{(v+1)}[\omega]= \\
& -\delta_{v 0} \rho_{S, I}(0)-\sum_{k=1}^{K} n_{k} \Theta_{k}(t) \rho_{n_{1}, \cdots, n_{k}-1, \cdots, n_{K}}^{(v-1)}[\omega] \\
& -i \sum_{k=1}^{K} n_{k} \gamma_{k} \rho_{n_{1}, \cdots, n_{K}}^{(v)}[\omega] .
\end{aligned}
$$

$\delta$ is the Kronecker symbol ( $\delta_{v 0}=1$ if $v=0,0$ otherwise).

For auxiliary matrices, we assume that for all $v$ and vector $\left\{n_{1}, \cdots, n_{K}\right\}, \rho_{n_{1}, \cdots, n_{K}}^{(v)}(0)=0$.

With an inverse Fourier-Laplace transform ( for a differentiable function $\left.f, \int_{0}^{+\infty} d t e^{-i \omega t} \frac{d}{d t} f(t)=i \omega f[\omega]-f(0)\right)$, this leads to the set of hierarchical equations of motion:

$$
\left\{\begin{array}{c}
\dot{\rho}_{S, I}(t)=\sum_{k=1}^{K} \Phi_{j}(t) \rho_{0_{1}, \cdots, 0_{k}+1, \cdots, 0_{K}}^{(1)}(t) \\
\dot{\rho}_{n_{1}, \cdots, n_{K}}^{(v)}(t)=\sum_{k=1}^{K} \Phi_{j}(t) \rho_{n_{1}, \cdots, n_{k}+1, \cdots, n_{K}}^{(v+1)}(t) \\
\quad+\sum_{k=1}^{K} n_{k} \Theta_{k}(t) \rho_{n_{1}, \cdots, n_{k}-1, \cdots, n_{K}}^{(v-1)}(t) \\
+i \sum_{k=1}^{K} n_{k} \gamma_{k} \rho_{n_{1}, \cdots, n_{K}}^{(v)}(t)
\end{array}\right.
$$

${ }^{1}$ G. Herzberg and H. C. Longuet-Higgins, "Intersection of potential energy surfaces in polyatomic molecules," Discuss Faraday Soc, vol. 35, no. 0, pp. 77-82, 1963.

${ }^{2}$ J. Michl and V. Bonacićic-Koutecký, Electronic Aspects Of Organic Photochemistry. Wiley, New York, 1990.

${ }^{3} \mathrm{M}$. Klessinger and J. Michl, Excited States and Photochemistry of Organic Molecules. Cambridge VCH, New York, 1995.

${ }^{4}$ D. R. Yarkony, "Diabolical conical intersections," Rev. Mod. Phys., vol. 68, pp. 985-1013, Oct. 1996.

${ }^{5}$ W. Domcke, D. Yarkony, and H. Koppel, Conical Intersection, Electronic Structure, Dynamics and Spectroscopy. World Scientific Publishing Co Pte Ltd, 2004.

${ }^{6}$ G. A. Worth and L. S. Cederbaum, "Beyond Born-Oppenheimer: Molecular dynamics through a conical intersection," Аппи. Rev. Phys. Chem., vol. 55, no. 1, pp. 127-158, 2004.

${ }^{7}$ I. Bersuker, The Jahn-Teller Effect. Cambridge University Press, Cambridge, 2006.

${ }^{8}$ M. Baer, Beyond Born-Oppenheimer: Electronic Nonadiabatic Couling Terms and Conical Intersections. N.J. Hoboken, Wiley, 2006.

${ }^{9}$ S. Matsika and P. Krause, "Nonadiabatic events and conical intersections," Annu. Rev. Phys. Chem., vol. 62, no. 1, pp. 621-643, 2011.

${ }^{10} \mathrm{~W}$. Domcke, D. Yarkony, and H. Koppel, Conical Intersection: Theory, Computation and Experiment. N.J. Hackensack, World Scientific, 2011.
${ }^{11}$ J. von Neuman and E. Wigner, "Uber merkwürdige diskrete Eigenwerte. Uber das Verhalten von Eigenwerten bei adiabatischen Prozessen," Physikalische Zeitschrift, vol. 30, pp. 467-470, Jan 1929.

${ }^{12}$ M. Kowalewski, B. P. Fingerhut, K. E. Dorfman, K. Bennett, and S. Mukamel, "Simulating coherent multidimensional spectroscopy of nonadiabatic molecular processes: From the infrared to the x-ray regime," Chem. Rev., vol. 117, no. 19, pp. 12165-12226, 2017.

${ }^{13}$ M. S. Schuurman and A. Stolow, "Dynamics at conical intersections," Annu. Rev. Phys. Chem., vol. 69, no. 1, pp. 427-450, 2018.

${ }^{14}$ E. C. Wu, Q. Ge, E. A. Arsenault, N. H. C. Lewis, N. L. Gruenke, M. J. Head-Gordon, and G. R. Fleming, "Two-dimensional electronicvibrational spectroscopic study of conical intersection dynamics: An experimental and electronic structure study," Phys. Chem. Chem. Phys., vol. 21, no. 26, pp. 14153-14163, 2019.

${ }^{15}$ E. Palacino-González, M. F. Gelin, and W. Domcke, "Analysis of transient-absorption pump-probe signals of nonadiabatic dissipative systems: "Ideal" and "real" spectra," J. Chem. Phys., vol. 150, no. 20, p. 204102, 2019.

${ }^{16}$ M. Garavelli, F. Bernardi, M. Olivucci, T. Vreven, S. Klein, P. Celani, and M. A. Robb, "Potential-energy surfaces for ultrafast photochemistry Static and dynamic aspects," Faraday Discuss, vol. 110, no. 0, pp. 51-70, 1998.

${ }^{17}$ M. A. Robb, M. Garavelli, M. Olivucci, and F. Bernardi, "A computational strategy for organic photochemistry," in Reviews in Computational Chemistry, pp. 87-146, John Wiley \& Sons, Ltd, 2007.

${ }^{18}$ M. Olivucci, Computational Potochemistry. Elsevier, Amsterdam, 2005.

${ }^{19}$ G. A. Worth, H.-D. Meyer, H. Köppel, L. S. Cederbaum, and I. Burghardt, "Using the MCTDH wavepacket propagation method to describe multimode non-adiabatic dynamics," Int. Rev. Phys. Chem., vol. 27, no. 3, pp. 569-606, 2008.

${ }^{20}$ B. Lasorne, G. A. Worth, and M. A. Robb, "Excited-state dynamics," Wiley Interdiscip. Rev. Comput. Mol. Sci., vol. 1, no. 3, pp. 460-475, 2011.

${ }^{21}$ M. A. Robb, "Chapter three - in this molecule there must be a conical intersection," Adv. Phys. Org. Chem., vol. 48, pp. 189 - 228, 2014.

${ }^{22}$ F. Gatti, B. Lasorne, H.-D. Meyer, and A. Nauts, Applications of Quantum Dynamics in Chemistry. Cham, Springer International Publishing, 2017.

${ }^{23}$ Y. Guan, H. Guo, and D. R. Yarkony, "Neural network based quasi-diabatic Hamiltonians with symmetry adaptation and a correct description of conical intersections," J. Chem. Phys., vol. 150, no. 21, p. 214101, 2019.

${ }^{24}$ M. Desouter-Lecomte, C. Galloy, J. C. Lorquet, and M. V. Pires, "Nonadiabatic interactions in unimolecular decay. V. Conical and Jahn-Teller intersections," J. Chem. Phys., vol. 71, no. 9, pp. 3661-3672, 1979.

${ }^{25}$ T. Van Voorhis, T. Kowalczyk, B. Kaduk, L.-P. Wang, C.-L. Cheng, and $\mathrm{Q}$. Wu, "The diabatic picture of electron transfer, reaction barriers, and molecular dynamics," Аnnu. Rev. Phys. Chem., vol. 61, no. 1, pp. 149$170,2010$. 
${ }^{26}$ C. A. Mead and D. G. Truhlar, "Conditions for the definition of a strictly diabatic electronic basis for molecular systems," J. Chem. Phys., vol. 77, no. 12 , pp. 6090-6098, 1982

${ }^{27}$ A. Thiel and H. Köppel, "Proposal and numerical test of a simple diabatization scheme," J. Chem. Phys., vol. 110, no. 19, pp. 9371-9383, 1999.

${ }^{28}$ E. R. Davidson, "Global topology of triatomic potential surfaces," J. Am. Chem. Soc., vol. 99, no. 2, pp. 397-402, 1977.

${ }^{29}$ M. Desouter-Lecomte, D. Dehareng, B. Leyh-Nihant, M. T. Praet, A. J. Lorquet, and J. C. Lorquet, "Nonadiabatic unimolecular reactions of polyatomic molecules," J. Phys. Chem., vol. 89, no. 2, pp. 214-222, 1985.

${ }^{30}$ G. J. Atchity, S. S. Xantheas, and K. Ruedenberg, "Potential energy surfaces near intersections," J. Chem. Phys., vol. 95, no. 3, pp. 1862-1876, 1991.

${ }^{31}$ B. Gonon, B. Lasorne, G. Karras, L. Joubert-Doriol, D. Lauvergnat, F. Billard, B. Lavorel, O. Faucher, S. Guérin, E. Hertz, and F. Gatti, "A generalized vibronic-coupling Hamiltonian for molecules without symmetry: Application to the photoisomerization of benzopyran," J. Chem. Phys., vol. 150 , no. 12, p. 124109, 2019.

${ }^{32}$ H. Wang and H.-D. Meyer, "On regularizing the ML-MCTDH equations of motion," J. Chem. Phys., vol. 149, no. 4, p. 044119, 2018.

${ }^{33}$ M. Ben-Nun and T. J. Martínez, "Ab initio quantum molecular dynamics," in Advances in Chemical Physics, pp. 439-512, John Wiley \& Sons, Ltd, 2002.

${ }^{34}$ B. G. Levine and T. J. Martínez, "Isomerization through conical intersections," Annu. Rev. Phys. Chem., vol. 58, no. 1, pp. 613-634, 2007.

${ }^{35}$ G. Richings, I. Polyak, K. Spinlove, G. Worth, I. Burghardt, and B. Lasorne, "Quantum dynamics simulations using Gaussian wavepackets: The vMCG method," Int. Rev. Phys. Chem., vol. 34, no. 2, pp. 269-308, 2015.

${ }^{36}$ F. A. Schroder, D. H. Turban, A. J. Musser, N. D. Hine, and A. W. Chin, "Tensor network simulation of multi-environmental open quantum dynamics via machine learning and entanglement renormalisation," Nat. Commun., vol. 10, no. 1, p. 1062, 2019.

${ }^{37}$ T. Schaupp and V. Engel, "A classical ride through a conical intersection," J. Chem. Phys., vol. 150, no. 3, p. 034301, 2019.

${ }^{38}$ J. C. Tully, "Mixed quantum-classical dynamics," Faraday Discuss, vol. 110 , no. 0, pp. 407-419, 1998.

${ }^{39}$ W. Xie, M. Sapunar, N. Došlić, M. Sala, and W. Domcke, "Assessing the performance of trajectory surface hopping methods: Ultrafast internal conversion in pyrazine," The Journal of Chemical Physics, vol. 150, no. 15, p. 154119, 2019.

${ }^{40} \mathrm{I}$. de Vega and D. Alonso, "Dynamics of non-Markovian open quantum systems," Rev. Mod. Phys., vol. 89, p. 015001, Jan. 2017.

${ }^{41}$ Y. Ke and Y. Zhao, "Hierarchy of stochastic Schrödinger equation towards the calculation of absorption and circular dichroism spectra," J. Chem. Phys., vol. 146, no. 17, p. 174105, 2017.

${ }^{42}$ Y.-C. Wang, Y. Ke, and Y. Zhao, "The hierarchical and perturbative forms of stochastic Schrödinger equations and their applications to carrier dynamics in organic materials," Wiley Interdiscip. Rev. Comput. Mol. Sci., vol. 9, no. 1, p. e1375, 2019.

${ }^{43} \mathrm{~K}$. Nakamura and Y. Tanimura, "Hierarchical Schrödinger equations of motion for open quantum dynamics," Phys Rev A, vol. 98, p. 012109, July 2018.

${ }^{44} \mathrm{~T}$. Ikeda and Y. Tanimura, "Probing photoisomerization processes by means of multi-dimensional electronic spectroscopy: The multi-state quantum hierarchical fokker-planck equation approach," The Journal of Chemical Physics, vol. 147, no. 1, p. 014102, 2017.

${ }^{45} \mathrm{~T}$. Ikeda and Y. Tanimura, "Phase-space wavepacket dynamics of internal conversion via conical intersection: Multi-state quantum Fokker-Planck equation approach," Chem. Phys., vol. 515, pp. 203 - 213, 2018. Ultrafast Photoinduced Processes in Polyatomic Molecules:Electronic Structure, Dynamics and Spectroscopy (Dedicated to Wolfgang Domcke on the occasion of his 70th birthday).

${ }^{46} \mathrm{~T}$. Ikeda and Y. Tanimura, "Low-temperature quantum fokker-planck and smoluchowski equations and their extension to multistate systems," Journal of Chemical Theory and Computation, vol. 15, no. 4, pp. 2517-2534, 2019. PMID: 30776312.

${ }^{47}$ T. Ikeda, A. G. Dijkstra, and Y. Tanimura, "Modeling and analyzing a photo-driven molecular motor system: Ratchet dynamics and non-linear optical spectra," The Journal of Chemical Physics, vol. 150, no. 11, p. $114103,2019$.
${ }^{48}$ Y. Tanimura and R. Kubo, "Time Evolution of a Quantum System in Contact with a Nearly Gaussian-Markoffian Noise Bath," J. Phys. Soc. Jpn., vol. 58, pp. 101-114, Jan. 1989.

${ }^{49}$ Y. Tanimura, "Stochastic Liouville, Langevin, Fokker-Planck, and master equation approaches to quantum dissipative systems," J. Phys. Soc. Jpn., vol. 75 , no. 8, p. $082001,2006$.

${ }^{50}$ A. M. Levine, M. Shapiro, and E. Pollak, "Hamiltonian theory for vibrational dephasing rates of small molecules in liquids," The Journal of Chemical Physics, vol. 88, no. 3, pp. 1959-1966, 1988.

${ }^{51}$ L. S. Cederbaum, E. Gindensperger, and I. Burghardt, "Short-time dynamics through conical intersections in macrosystems," Phys. Rev. Lett., vol. 94, p. 113003, Mar. 2005.

${ }^{52}$ E. Gindensperger, I. Burghardt, and L. S. Cederbaum, "Short-time dynamics through conical intersections in macrosystems. I. Theory: Effectivemode formulation," J. Chem. Phys., vol. 124, no. 14, p. 144103, 2006.

${ }^{53} \mathrm{~K}$. H. Hughes, C. D. Christ, and I. Burghardt, "Effective-mode representation of non-Markovian dynamics: A hierarchical approximation of the spectral density. II. Application to environment-induced nonadiabatic dynamics," J. Chem. Phys., vol. 131, no. 12, p. 124108, 2009.

${ }^{54}$ A. Pereverzev, E. R. Bittner, and I. Burghardt, "Energy and chargetransfer dynamics using projected modes," J. Chem. Phys., vol. 131, no. 3 , p. 034104, 2009.

${ }^{55}$ R. Martinazzo, K. H. Hughes, and I. Burghardt, "Unraveling a Brownian particle's memory with effective mode chains," Phys Rev E, vol. 84, p. 030102, Sept. 2011.

${ }^{56}$ J. Roden, W. T. Strunz, K. B. Whaley, and A. Eisfeld, "Accounting for intra-molecular vibrational modes in open quantum system description of molecular systems," J. Chem. Phys., vol. 137, no. 20, p. 204110, 2012.

${ }^{57}$ A. Garg, J. N. Onuchic, and V. Ambegaokar, "Effect of friction on electron transfer in biomolecules," J. Chem. Phys., vol. 83, no. 9, pp. 4491-4503, 1985.

${ }^{58}$ Y. Tanimura and S. Mukamel, "Optical stark spectroscopy of a brownian oscillator in intense fields," Journal of the Physical Society of Japan, vol. 63 , no. 1 , pp. $66-77,1994$

${ }^{59} \mathrm{~V}$. Chernyak and S. Mukamel, "Collective coordinates for nuclear spectral densities in energy transfer and femtosecond spectroscopy of molecular aggregates," The Journal of Chemical Physics, vol. 105, no. 11, pp. 45654583, 1996

${ }^{60}$ J. Cao, "A phase-space study of Bloch-Redfield theory," J. Chem. Phys., vol. 107, no. 8, pp. 3204-3209, 1997.

${ }^{61}$ J. Cao and G. A. Voth, "A unified framework for quantum activated rate processes. II. The nonadiabatic limit," J. Chem. Phys., vol. 106, no. 5, pp. 1769-1779, 1997.

${ }^{62}$ M. Thoss, H. Wang, and W. H. Miller, "Self-consistent hybrid approach for complex systems: Application to the spin-boson model with Debye spectral density," J. Chem. Phys., vol. 115, no. 7, pp. 2991-3005, 2001.

${ }^{63} \mathrm{M}$. Tanaka and Y. Tanimura, "Quantum dissipative dynamics of electron transfer reaction system: Nonperturbative hierarchy equations approach," Journal of the Physical Society of Japan, vol. 78, no. 7, p. 073802, 2009.

${ }^{64} \mathrm{M}$. Tanaka and Y. Tanimura, "Multistate electron transfer dynamics in the condensed phase: Exact calculations from the reduced hierarchy equations of motion approach," J. Chem. Phys., vol. 132, no. 21, p. 214502, 2010.

${ }^{65}$ A. Chenel, E. Mangaud, I. Burghardt, C. Meier, and M. Desouter-Lecomte, "Exciton dissociation at donor-acceptor heterojunctions: Dynamics using the collective effective mode representation of the spin-boson model," $J$. Chem. Phys., vol. 140, no. 4, p. 044104, 2014.

${ }^{66}$ J. Iles-Smith, N. Lambert, and A. Nazir, "Environmental dynamics, correlations, and the emergence of noncanonical equilibrium states in open quantum systems," Phys. Rev. A, vol. 90, p. 032114, Sept. 2014.

${ }^{67}$ J. Iles-Smith, A. G. Dijkstra, N. Lambert, and A. Nazir, "Energy transfer in structured and unstructured environments: Master equations beyond the Born-Markov approximations," J. Chem. Phys., vol. 144, no. 4, p. 044110 , 2016.

${ }^{68}$ M. Wertnik, A. Chin, F. Nori, and N. Lambert, "Optimizing co-operative multi-environment dynamics in a dark-state-enhanced photosynthetic heat engine," J. Chem. Phys., vol. 149, no. 8, p. 084112, 2018.

${ }^{69}$ A. Kühl and W. Domcke, "Multilevel Redfield description of the dissipative dynamics at conical intersections," J. Chem. Phys., vol. 116, no. 1, pp. 263-274, 2002.

${ }^{70}$ D.-L. Qi, H.-G. Duan, Z.-R. Sun, R. J. D. Miller, and M. Thorwart, “Track- 
ing an electronic wave packet in the vicinity of a conical intersection," $J$. Chem. Phys., vol. 147, no. 7, p. 074101, 2017.

${ }^{71}$ A. J. Schile and D. T. Limmer, "Simulating conical intersection dynamics in the condensed phase with hybrid quantum master equations," J. Chem. Phys., vol. 151, no. 1, p. 014106, 2019.

${ }^{72}$ L. Chen, M. F. Gelin, V. Y. Chernyak, W. Domcke, and Y. Zhao, "Dissipative dynamics at conical intersections: Simulations with the hierarchy equations of motion method," Faraday Discuss, vol. 194, no. 0, pp. 61-80, 2016.

${ }^{73}$ L. Chen, M. F. Gelin, Y. Zhao, and W. Domcke, "Mapping of wave packet dynamics at conical intersections by time- and frequency-resolved fluorescence spectroscopy: A computational study," The Journal of Physical Chemistry Letters, vol. 10, no. 19, pp. 5873-5880, 2019. PMID: 31518141.

${ }^{74}$ H.-G. Duan and M. Thorwart, "Quantum mechanical wave packet dynamics at a conical intersection with strong vibrational dissipation," J. Phys. Chem. Lett., vol. 7, no. 3, pp. 382-386, 2016.

${ }^{75}$ A. G. Dijkstra and V. I. Prokhorenko, "Simulation of photo-excited adenine in water with a hierarchy of equations of motion approach," J. Chem. Phys., vol. 147, no. 6, p. 064102, 2017.

${ }^{76}$ L. Zhu, H. Liu, W. Xie, and Q. Shi, "Explicit system-bath correlation calculated using the hierarchical equations of motion method," J. Chem. Phys., vol. 137, no. 19, p. 194106, 2012.

${ }^{77}$ H. Liu, L. Zhu, S. Bai, and Q. Shi, "Reduced quantum dynamics with arbitrary bath spectral densities: Hierarchical equations of motion based on several different bath decomposition schemes," J. Chem. Phys., vol. 140, no. 13, p. 134106, 2014.

${ }^{78}$ H. Choi, W.-J. Son, S. Shin, B. Y. Chang, and I. R. Sola, "Selective photodissociation in diatomic molecules by dynamical Stark-shift control," $J$. Chem. Phys., vol. 128, no. 10, p. 104315, 2008.

${ }^{79}$ S. Scheit, Y. Arasaki, and K. Takatsuka, "Control scheme of nonadiabatic transitions with the dynamical shift of potential curve crossing," J. Chem. Phys., vol. 140, no. 24, p. 244115, 2014.

${ }^{80}$ M. Saab, M. Sala, B. Lasorne, F. Gatti, and S. Guérin, "Full-dimensional control of the radiationless decay in pyrazine using the dynamic Stark effect," J. Chem. Phys., vol. 141, no. 13, p. 134114, 2014.

${ }^{81}$ M. Richter, F. Bouakline, J. González-Vázquez, L. Martínez-Fernández, I. Corral, S. Patchkovskii, F. Morales, M. Ivanov, F. Martín, and O. Smirnova, "Sub-laser-cycle control of coupled electron-nuclear dynamics at a conical intersection," New J. Phys., vol. 17, p. 113023, nov 2015.

${ }^{82}$ B. G. Levine, M. P. Esch, B. S. Fales, D. T. Hardwick, W.-T. Peng, and Y. Shu, "Conical intersections at the nanoscale: Molecular ideas for materials," Аnnu. Rev. Phys. Chem., vol. 70, no. 1, pp. 21-43, 2019.

${ }^{83} \mathrm{E}$. Pollak, "Theory of activated rate processes: A new derivation of Kramers' expression,' J. Chem. Phys., vol. 85, no. 2, pp. 865-867, 1986.

${ }^{84} \mathrm{Y}$. Tanimura, "Reduced hierarchy equations of motion approach with Drude plus Brownian spectral distribution: Probing electron transfer processes by means of two-dimensional correlation spectroscopy," J. Chem. Phys., vol. 137, no. 22, p. 22A550, 2012.

${ }^{85}$ R. Martinazzo, K. H. Hughes, F. Martelli, and I. Burghardt, "Effective spectral densities for system-environment dynamics at conical intersections: S2-S1 conical intersection in pyrazine," Chem. Phys., vol. 377, no. 1 , pp. $21-29,2010$.

${ }^{86} \mathrm{H}$. Tamura, R. Martinazzo, M. Ruckenbauer, and I. Burghardt, "Quantum dynamics of ultrafast charge transfer at an oligothiophene-fullerene heterojunction," J. Chem. Phys., vol. 137, no. 22, p. 22A540, 2012.

${ }^{87}$ S. Valleau, A. Eisfeld, and A. Aspuru-Guzik, "On the alternatives for bath correlators and spectral densities from mixed quantum-classical simulations," J. Chem. Phys., vol. 137, no. 22, p. 224103, 2012.

${ }^{88}$ M. Aghtar, J. Strümpfer, C. Olbrich, K. Schulten, and U. Kleinekathöfer, "Different types of vibrations interacting with electronic excitations in phycoerythrin 545 and Fenna-Matthews-Olson antenna systems," J. Phys. Chem. Lett., vol. 5, no. 18, pp. 3131-3137, 2014.

${ }^{89} \mathrm{H}$. Wang, M. Thoss, and W. H. Miller, "Systematic convergence in the dynamical hybrid approach for complex systems: A numerically exact methodology," J. Chem. Phys., vol. 115, no. 7, pp. 2979-2990, 2001.

${ }^{90} \mathrm{C}$. Meier and D. J. Tannor, "Non-Markovian evolution of the density operator in the presence of strong laser fields," J. Chem. Phys., vol. 111, no. 8, pp. 3365-3376, 1999.
${ }^{91}$ R. Martinazzo, B. Vacchini, K. H. Hughes, and I. Burghardt, "Communication: Universal markovian reduction of brownian particle dynamics," $J$. Chem. Phys., vol. 134, no. 1, p. 011101, 2011.

${ }^{92}$ A. Pomyalov, C. Meier, and D. J. Tannor, "The importance of initial correlations in rate dynamics: A consistent non-Markovian master equation approach," Chem. Phys., vol. 370, pp. 98-108, May 2010.

${ }^{93}$ R. Kubo, "Stochastic Liouville Equations," J. Math. Phys., vol. 4, no. 2, p. $174,1963$.

${ }^{94}$ A. Ishizaki and Y. Tanimura, "Quantum Dynamics of System Strongly Coupled to Low-Temperature Colored Noise Bath: Reduced Hierarchy Equations Approach,” J. Phys. Soc. Jpn., vol. 74, pp. 3131-3134, Dec. 2005.

${ }^{95}$ Q. Shi, L. Chen, G. Nan, R.-X. Xu, and Y. Yan, "Efficient hierarchical Liouville space propagator to quantum dissipative dynamics," J. Chem. Phys., vol. 130, no. 8, p. 084105, 2009.

${ }^{96}$ J. Jin, S. Welack, J. Luo, X.-Q. Li, P. Cui, R.-X. Xu, and Y. Yan, "Dynamics of quantum dissipation systems interacting with fermion and boson grand canonical bath ensembles: Hierarchical equations of motion approach," J. Chem. Phys., vol. 126, no. 13, p. 134113, 2007.

${ }^{97}$ J. Hu, M. Luo, F. Jiang, R.-X. Xu, and Y. Yan, "Padé spectrum decompositions of quantum distribution functions and optimal hierarchical equations of motion construction for quantum open systems," J. Chem. Phys., vol. 134, no. 24, p. 244106, 2011.

${ }^{98}$ L. Cui, H.-D. Zhang, X. Zheng, R.-X. Xu, and Y. Yan, "Highly efficient and accurate sum-over-poles expansion of Fermi and Bose functions at near zero temperatures: Fano spectrum decomposition scheme," J. Chem. Phys., vol. 151, no. 2, p. 024110, 2019.

${ }^{99} \mathrm{H}$. Rahman and U. Kleinekathöfer, "Chebyshev hierarchical equations of motion for systems with arbitrary spectral densities and temperatures," $J$. Chem. Phys., vol. 150, no. 24, p. 244104, 2019.

${ }^{100} \mathrm{~A}$. Ishizaki and G. R. Fleming, "On the adequacy of the Redfield equation and related approaches to the study of quantum dynamics in electronic energy transfer," J. Chem. Phys., vol. 130, p. 234110, June 2009.

${ }^{101}$ A. Ishizaki, T. R. Calhoun, G. S. Schlau-Cohen, and G. R. Fleming, "Quantum coherence and its interplay with protein environments in photosynthetic electronic energy transfer," Phys. Chem. Chem. Phys., vol. 12, pp. 7319-7337, July 2010.

102 J. Strümpfer and K. Schulten, "Excited state dynamics in photosynthetic reaction center and light harvesting complex 1,"J. Chem. Phys., vol. 137, no. 6, p. 065101, 2012.

${ }^{103}$ S.-H. Yeh, J. Zhu, and S. Kais, "Population and coherence dynamics in light harvesting complex II (LH2)," J. Chem. Phys., vol. 137, no. 8, p. 084110, 2012.

${ }^{104}$ L. Chen, R. Zheng, Y. Jing, and Q. Shi, "Simulation of the twodimensional electronic spectra of the Fenna-Matthews-Olson complex using the hierarchical equations of motion method," J. Chem. Phys., vol. 134, no. 19 , p. $194508,2011$.

${ }^{105} \mathrm{~L}$. Song and Q. Shi, "Calculation of correlated initial state in the hierarchical equations of motion method using an imaginary time path integral approach," J. Chem. Phys., vol. 143, no. 19, p. 194106, 2015.

${ }^{106} \mathrm{E}$. Mangaud, C. Meier, and M. Desouter-Lecomte, "Analysis of the nonMarkovianity for electron transfer reactions in an oligothiophene-fullerene heterojunction," Chem. Phys., vol. 494, pp. 90 - 102, 2017.

${ }^{107}$ J.-J. Ding, R.-X. Xu, and Y. Yan, "Optimizing hierarchical equations of motion for quantum dissipation and quantifying quantum bath effects on quantum transfer mechanisms," J. Chem. Phys., vol. 136, no. 22, p. 224103, 2012.

${ }^{108}$ L. Song and Q. Shi, "Hierarchical equations of motion method applied to nonequilibrium heat transport in model molecular junctions: Transient heat current and high-order moments of the current operator," Phys. Rev. B, vol. 95, p. 064308, Feb. 2017.

${ }^{109}$ Y. Yan, L. Song, and Q. Shi, "Understanding the free energy barrier and multiple timescale dynamics of charge separation in organic photovoltaic cells," J. Chem. Phys., vol. 148, no. 8, p. 084109, 2018.

${ }^{110}$ A. G. Dijkstra and Y. Tanimura, "Non-markovian entanglement dynamics in the presence of system-bath coherence," Phys. Rev. Lett., vol. 104, p. 250401, June 2010.

${ }^{111}$ Y. Tanimura, "Reduced hierarchical equations of motion in real and imaginary time: Correlated initial states and thermodynamic quantities," $J$. Chem. Phys., vol. 141, no. 4, p. 044114, 2014. 
${ }^{112}$ A. Chin, E. Mangaud, V. Chevet, O. Atabek, and M. Desouter-Lecomte, "Visualising the role of non-perturbative environment dynamics in the dissipative generation of coherent electronic motion," Chem. Phys., vol. 525, p. $110392,2019$.

${ }^{113}$ Q. Shi, L. Chen, G. Nan, R. Xu, and Y. Yan, "Electron transfer dynamics: Zusman equation versus exact theory," J. Chem. Phys., vol. 130, no. 16, p. $164518,2009$.

114 Á. Rivas, S. F. Huelga, and M. B. Plenio, "Quantum non-Markovianity: Characterization, quantification and detection," Rep. Prog. Phys., vol. 77, p. 094001, Aug. 2014.

${ }^{115}$ H.-P. Breuer, E.-M. Laine, J. Piilo, and B. Vacchini, "Colloquium: NonMarkovian dynamics in open quantum systems," Rev Mod Phys, vol. 88, p. 021002, Apr. 2016.

${ }^{116}$ H.-P. Breuer and F. Petruccione, The Theory of Open Quantum Systems. Oxford University Press, 2002.

${ }^{117}$ S. Lorenzo, F. Plastina, and M. Paternostro, "Role of environmental correlations in the non-Markovian dynamics of a spin system," Phys Rev A, vol. 84, p. 032124, Sept. 2011.

${ }^{118}$ M. J. W. Hall, J. D. Cresser, L. Li, and E. Andersson, "Canonical form of master equations and characterization of non-Markovianity," Phys Rev A, vol. 89, p. 042120, Apr. 2014.

${ }^{119}$ G. Kimura, "The Bloch vector for N-level systems," Phys. Lett. A, vol. 314, no. 5 , pp. $339-349,2003$.

${ }^{120}$ D. Aerts and M. S. de Bianchi, "The extended Bloch representation of quantum mechanics and the hidden-measurement solution to the measurement problem," Ann. Phys., vol. 351, pp. 975 - 1025, 2014.

${ }^{121}$ H.-S. Zeng, N. Tang, Y.-P. Zheng, and G.-Y. Wang, "Equivalence of the measures of non-markovianity for open two-level systems," Phys. Rev. A, vol. 84, p. 032118, Sep 2011.

${ }^{122}$ G. Guarnieri, A. Smirne, and B. Vacchini, "Quantum regression theorem and non-markovianity of quantum dynamics," Phys. Rev. A, vol. 90, p. 022110, Aug 2014.

123 "Supporting information,"

${ }^{124}$ William H. Press, Saul A. Teukolsky, William T. Vetterling, and Brian P. Flannery, Numerical Recipes - The Art of Scientific Computing. Cambridge University Press, 1992.

${ }^{125} \mathrm{~J}$. Alvarellos and H. Metiu, "The evolution of the wave function in a curve crossing problem computed by a fast Fourier transform method," J. Chem. Phys., vol. 88, no. 8, pp. 4957-4966, 1988.

${ }^{126}$ P. Nalbach, S. Javanbakht, C. Stahl, and M. Thorwart, "Stueckelberg oscillations in a two-state two-path model of a conical intersection," Ann. Phys., vol. 529, no. 9, p. 1600147, 2017.
${ }^{127}$ C. Kreisbeck, T. Kramer, and A. Aspuru-Guzik, "Scalable highperformance algorithm for the simulation of exciton dynamics. application to the light-harvesting complex II in the presence of resonant vibrational modes," J. Chem. Theory Comput., vol. 10, no. 9, pp. 4045-4054, 2014.

${ }^{128} \mathrm{P}$. Rebentrost and A. Aspuru-Guzik, "Communication: Exciton-phonon information flow in the energy transfer process of photosynthetic complexes," J. Chem. Phys., vol. 134, no. 10, p. 101103, 2011.

${ }^{129}$ R. Kosloff, "Quantum thermodynamics and open-systems modeling," $J$. Chem. Phys., vol. 150, no. 20, p. 204105, 2019.

${ }^{130}$ P. Strasberg, G. Schaller, N. Lambert, and T. Brandes, "Nonequilibrium thermodynamics in the strong coupling and non-Markovian regime based on a reaction coordinate mapping," New J. Phys., vol. 18, p. 073007, July 2016.

${ }^{131}$ P. V. Demekhin and L. S. Cederbaum, "Light-induced conical intersections in polyatomic molecules: General theory, strategies of exploitation, and application," The Journal of Chemical Physics, vol. 139, no. 15, p. 154314, 2013.

${ }^{132}$ T. Szidarovszky, G. J. Halász, A. G. Császár, L. S. Cederbaum, and g. Vibók, "Conical intersections induced by quantum light: Field-dressed spectra from the weak to the ultrastrong coupling regimes," J.Phys. Chem. Lett., vol. 9, no. 21, pp. 6215-6223, 2018.

${ }^{133}$ E. Mangaud, R. Puthumpally-Joseph, D. Sugny, C. Meier, O. Atabek, and M. Desouter-Lecomte, "Non-markovianity in the optimal control of an open quantum system described by hierarchical equations of motion," New Journal of Physics, vol. 20, p. 043050, apr 2018.

${ }^{134} \mathrm{~A}$. Ishizaki and G. R. Fleming, "Unified treatment of quantum coherent and incoherent hopping dynamics in electronic energy transfer: Reduced hierarchy equation approach," J. Chem. Phys., vol. 130, no. 23, p. 234111, 2009.

${ }^{135}$ N. G. Van Kampen, Stochastic Processes in Physics and Chemistry. Elsevier, Nov. 1992.

${ }^{136}$ T. Takagahara, E. Hanamura, and R. Kubo, "Stochastic Models of Intermediate State Interaction in Second Order Optical Processes -Stationary Response. II-," J. Phys. Soc. Jpn., vol. 43, pp. 811-816, Sept. 1977.

${ }^{137}$ V. Chernyak and S. Mukamel, "Collective coordinates for nuclear spectral densities in energy transfer and femtosecond spectroscopy of molecular aggregates," J. Chem. Phys., vol. 105, no. 11, pp. 4565-4583, 1996.

${ }^{138}$ M. Wertnik, A. Chin, F. Nori, and N. Lambert, "Optimizing co-operative multi-environment dynamics in a dark-state-enhanced photosynthetic heat engine," J. Chem. Phys., vol. 149, no. 8, p. 084112, 2018. 